Article

\title{
An Efficient and Lightweight Convolutional Neural Network for Remote Sensing Image Scene Classification
}

\author{
Donghang Yu®, Qing Xu *, Haitao Guo, Chuan Zhao, Yuzhun Lin and Daoji Li® \\ Institute of Geospatial Information, PLA Strategic Support Force Information Engineering University, \\ Zheng Zhou 450001, China; dong_hang@aliyun.com (D.Y.); ghtgjp2002@163.com (H.G.); \\ hehe549124@outlook.com (C.Z.); lyz120218@163.com (Y.L.); wang111@alumni.sjtu.edu.cn (D.L.) \\ * Correspondence: 13937169139@139.com
}

Received: 22 February 2020; Accepted: 1 April 2020; Published: 2 April 2020

\begin{abstract}
Classifying remote sensing images is vital for interpreting image content. Presently, remote sensing image scene classification methods using convolutional neural networks have drawbacks, including excessive parameters and heavy calculation costs. More efficient and lightweight CNNs have fewer parameters and calculations, but their classification performance is generally weaker. We propose a more efficient and lightweight convolutional neural network method to improve classification accuracy with a small training dataset. Inspired by fine-grained visual recognition, this study introduces a bilinear convolutional neural network model for scene classification. First, the lightweight convolutional neural network, MobileNetv2, is used to extract deep and abstract image features. Each feature is then transformed into two features with two different convolutional layers. The transformed features are subjected to Hadamard product operation to obtain an enhanced bilinear feature. Finally, the bilinear feature after pooling and normalization is used for classification. Experiments are performed on three widely used datasets: UC Merced, AID, and NWPU-RESISC45. Compared with other state-of-art methods, the proposed method has fewer parameters and calculations, while achieving higher accuracy. By including feature fusion with bilinear pooling, performance and accuracy for remote scene classification can greatly improve. This could be applied to any remote sensing image classification task.
\end{abstract}

Keywords: scene classification; remote sensing image; bilinear model; MobileNet; convolutional neural network

\section{Introduction}

In recent years, with the development of Earth observation technology, remote sensing image resolution has continuously improved, datasets have become larger, and applications have continued to expand. Therefore, rapid and efficient interpretation of these images has important applications [1-5].

Classification and scene recognition are important methods for remote sensing image interpretation. Scene classification refers to dividing the image into blocks and labeling each with an appropriate category (such as residential areas, farmland, rivers, and forests) according to the makeup of the blocks. This is helpful for image management, retrieval, analysis, detection, and recognition of typical targets. When resolution increases, images become more diverse, allowing for fine-grained classification and identification. At the same time, the details of high-resolution remote sensing images are richer, the features in the image are more diverse, and the objects on the ground are usually staggered. The similarity between images of the same type decreases while the difference of same types increases significantly [6,7]. In addition, it is necessary to consider rotation and positional relationship among targets in the image. These problems bring challenges to high-precision scene classification. 
At present, the classification methods with better performance can be broadly divided into two categories [3,4,8,9]: using handcrafted features and using deep learning.

1. Methods using handcrafted features. These methods mainly use handcrafted, low-level and middle-level features. Low-level features include color [10], gray-level co-occurrence matrix (GLCM) [11,12], local binary patterns (LBP) [13-16], texture features, Gabor descriptors [17], histogram of oriented graphic (HOG) features [18], and scale-invariant feature transform (SIFT) [19-21], etc. These features are easy to understand and implement, but cannot effectively represent high-level image semantic information. Using these features for image classification usually results in low accuracy. Therefore, low-level features are rarely used alone; they are usually used with middle-level features. The middle-level features are obtained by encoding the low-level features. This allows a relationship between the low-level features and the image semantic information to be established, improving classification performance. Bag of Visual Words (BOVW) is the most commonly adopted image classification method with middle-level features. Before the emergence of deep convolutional neural networks, BOVW had been the mainstream method for remote sensing image scene classification [22-27]. Spatial Pyramid Matching (SPM) [28], Spatial Co-occurrence Kernel (SCK) [29], Latent Dirichlet Allocation (LDA) [30,31], Probabilistic Latent Semantic Analysis (pLSA) [32], Fisher Kernel [33,34], Vector of Locally Aggregated Descriptors (VLAD) [35] and other technologies have also been widely used in remote sensing image scene classification for mining image semantic information. Compared with low-level features, scene classification methods using middle-level features have achieved higher accuracy. However, these methods require clever design features or specific constraints to increase feature discrimination. Many factors must be considered in feature design and their generalization is poor, especially in constructing middle-level features. The issues of information ambiguity and redundancy still lack effective solutions. The relationship between the middle-level features and semantic information has not been fully explored. It is hard for methods using handcrafted features to achieve high-precision image classification for complex scenes or massive data.

2. Methods using deep learning. Introducing deep learning, especially convolutional neural networks $(\mathrm{CNN})$, into remote sensing image scene classification has greatly improved accuracy and efficiency. Until now, there have been two main methods of using $\mathrm{CNN}$ for scene classification: 1) using the CNN pretrained on ImageNet [36] for fine-tuning [3,37-39] or feature extraction; 2) improving the structure of existing CNNs [8,40-46], loss functions [46-50], or combining CNNs with handcrafted features [51-55]. Chen et al. [3] fine-tuned VGG16 [56] and achieved a far higher classification accuracy than methods using handcrafted features on large-scale remote sensing image datasets. Nogueira et al. [38] adopted three strategies (training from scratch, fine-tuning, and utilizing CNNs as feature extractors) on multiple CNNs to classify remote sensing image scenes. The results showed that all three strategies can achieve high accuracy, especially the one using CNN as a feature extractor before using a linear SVM classifier to retrain the extracted features. Chaib et al. [57] utilized the discriminative correlation analysis method to fuse features extracted by the CNN and the overall classification accuracy reached $92.96 \%$ when the training ratio is $20 \%$. Cheng [47] and Goel [50], among others, used metric learning to improve feature distinguishability from different types of images. Zhu et al. [53] combined handcrafted features with high-level semantic features extracted by deep CNNs to improve the performance of scene classification, but this method cannot be trained and implemented in an end-to-end approach. Compared with traditional handcrafted features, training an existing deep CNN with remote sensing image data could achieve outstanding performance. Ameliorating the structure or loss function of CNNs also could improve classification accuracy. However, when considering a small training dataset (for example, when the training ratio is less than $20 \%$ ), there are still many challenges for producing fast and high-precision classification for more diverse scenes.

Most current high-precision scene classification methods adopt deep CNNs (such as VGG16, GoogLeNet [58], ResNet50 [59], etc.) or add handcrafted features to the features extracted by deep CNNs. Generally, a deep CNN has numerous parameters requiring super-computing power to reduce calculation time. A deep $\mathrm{CNN}$ has disadvantages such as training difficulties, low efficiency, and poor 
applicability. Due to the addition of handcrafted features, the classification pipeline is also divided into two stages, which cannot be trained or implemented in an end-to-end manner. There are still few methods available for remote sensing image scene classification and object detection (such as methods proposed by Zhang et al. [60], Zhang et al. [61] Teimouri et al. [62], etc.,) so far.

In view of the above problems, therefore, this study introduces the idea of feature fusion in the bilinear model $[63,64]$ with the CNN MobileNetv2 [65] and designs an efficient and lightweight $\mathrm{CNN}$ called BiMobileNet for remote sensing image classification. The architecture designed can not only achieve higher classification accuracy but also has fewer calculation numbers and parameters. The main contributions of this article are as follows:

(1) The idea of a bilinear model in fine-grained visual recognition is introduced into remote sensing image classification, which enhances the ability of the CNN to identify different scene types. Compared with the state-of-the-art methods for remote sensing image scene classification, the proposed method can obtain superior performance.

(2) By integrating the lightweight CNN MobileNetv2 and the feature fusion method of the bilinear model, the method in this study considers both the advantages of a lightweight structure and high accuracy. Compared with other state-of-the-art methods, the proposed architecture has fewer parameters and calculations. Therefore, image classification speed will be higher, rendering it more viable for production purposes and applications.

(3) This study proposes that both the accuracy and complexity of the method should be considered simultaneously during classification. The method should be evaluated comprehensively in three aspects: accuracy, parameter, and calculation. In addition, we find that most methods use the UC Merced dataset with a training ratio of $80 \%$, and the classification accuracy is close to saturation. We provide an accuracy benchmark when the training ratio is less than $30 \%$.

The remainder of this study is organized as follows. In Section 2, we illustrate the datasets used and the proposed architecture in detail. In Section 3, results and analysis of experiments on several datasets are detailed. Section 4 discusses results and Section 5 concludes the study with a summary of our method.

\section{Materials and Methods}

\subsection{Materials}

In order to verify the effectiveness of BiMobileNet in remote sensing image scene classification, three datasets (UC Merced [4], AID [2], and NWPU-RESISC45 [3]) were used.

The images in the UC Merced dataset are selected from aerial images in the U.S. Geological Survey (USGS) national city map. The dataset contains 21 scene types, such as farmland, residential area, forest, and oil tank. Each scene type consists of 100 images with a size of $256 \times 256$ pixels. In total, this dataset consists of 2100 RGB images with spatial resolution of $\sim 0.3 \mathrm{~m}$. Figure 1 shows examples from this dataset. More information on the dataset can be found at http://vision.ucmerced.edu/datasets. 


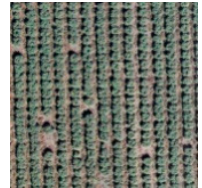

(1)

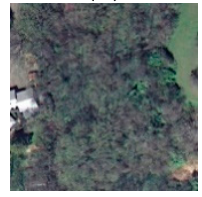

(8)

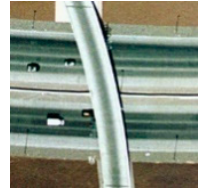

(15)

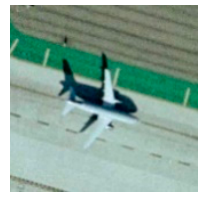

(2)

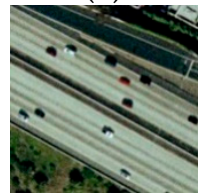

(9)

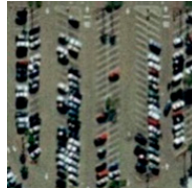

(16)

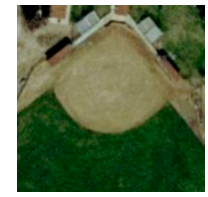

(3)

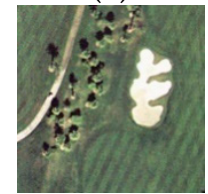

(10)

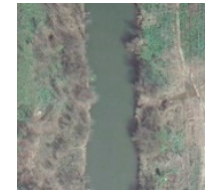

(17)

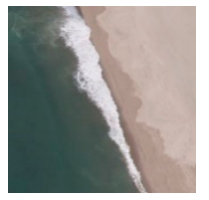

(4)

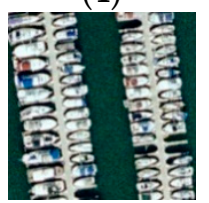

(11)

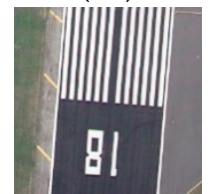

(18)

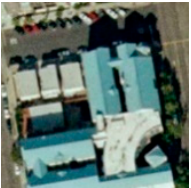

(5)

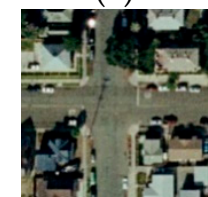

(12)

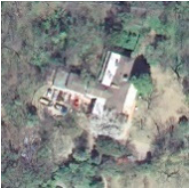

(19)

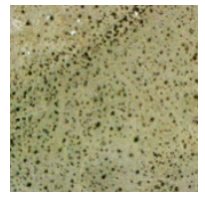

(6)

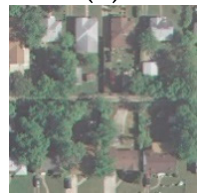

(13)

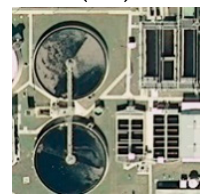

(20)

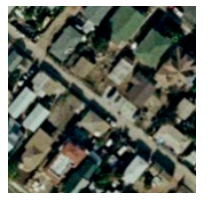

(7)

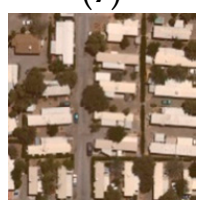

(14)

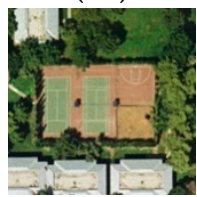

(21)

Figure 1. Class representatives of the UC Merced dataset: (1) agricultural; (2) airplane; (3) baseball diamond; (4) beach; (5) buildings; (6) chaparral; (7) dense residential; (8) forest; (9) freeway; (10) golf course; (11) harbor; (12) intersection; (13) medium residential; (14) mobile home park; (15) overpass; (16) parking lot; (17) river; (18) runway; (19) sparse residential; (20) storage tanks; and (21) tennis court.

Compared with the UC Merced dataset, the AID dataset extends the number of scene categories to 30 , with categories more finely classified. Each category contains $\sim 220$ to 440 RGB images; the total number of images in the dataset is 10,000. Image size is $600 \times 600$ pixels and the resolution is $\sim 0.5-8 \mathrm{~m}$. Figure 2 shows representative images of each class. More detailed information on this dataset can be found at http://www.lmars.whu.edu.cn/xia/AID-project.html.

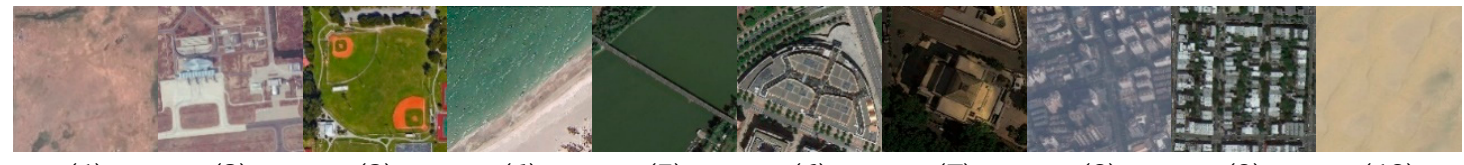

(1)

(2)

(3)

(4)

(5)

(6)

(8)

(9)

(10)

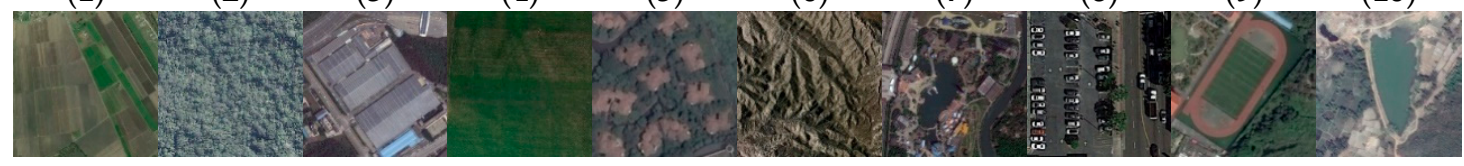
(11)
(12)
(13)
(14)
(15)
(16)
(17)
(18)
(19)
(20)

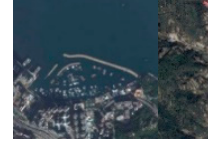
(21)
(22)
(23)
(24)
(25)
(26)
(27)
(28)
(29)
(30)

Figure 2. Class representatives of the AID dataset: (1) airport; (2) bareland; (3) baseball field; (4) beach; (5) bridge; (6) center; (7) church; (8) commercial; (9) dense residential; (10) desert; (11) farmland;

(12) forest; (13) industrial; industrial; (14) meadow; (15) medium residential; (16) mountain; (17) park;

(18) parking; (19) playground; (20) pond; (21) port; (22) railway station; (23) resort; (24) river; (25) school;

(26) sparse residential; (27) square; (28) stadium; (29) storage tanks; (30) viaduct.

NWPU-RESISC45 is a large-scale remote sensing image dataset, which further expands the number of categories and images. It contains 45 categories, each consisting of 700 RGB images with a size of $256 \times 256$ pixels. Image resolution ranges from $0.2-30 \mathrm{~m}$. In addition, images cover more than 100 countries and regions, including different weather, seasons, spatial resolution, and occlusion factors. Compared with other datasets, NWPU-RESISC45 images are more complex and diverse. 
Figure 3 shows representative images of each class. More detailed information can be found at http://www.escience.cn/people/JunweiHan/NWPU-RESISC45.html.

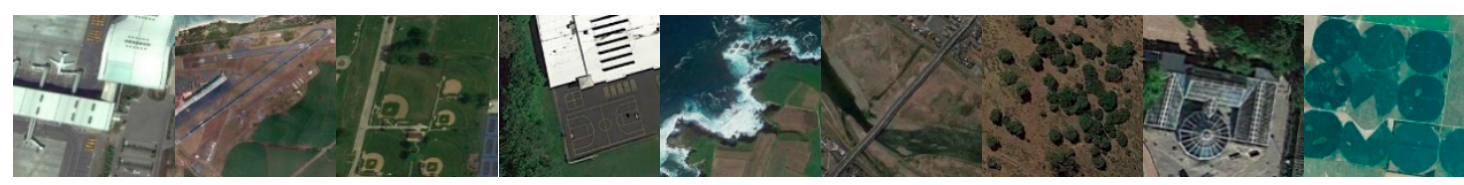

(1)

(2)

(3)

(4)

(5)

(6)

(7)

(8)

(9)

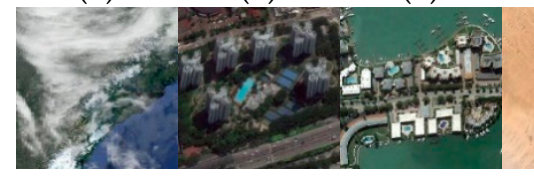

(10)

(11)

(12)

(13)
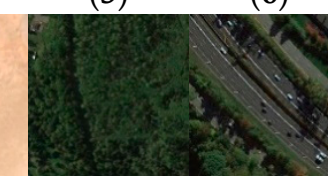

1) 0
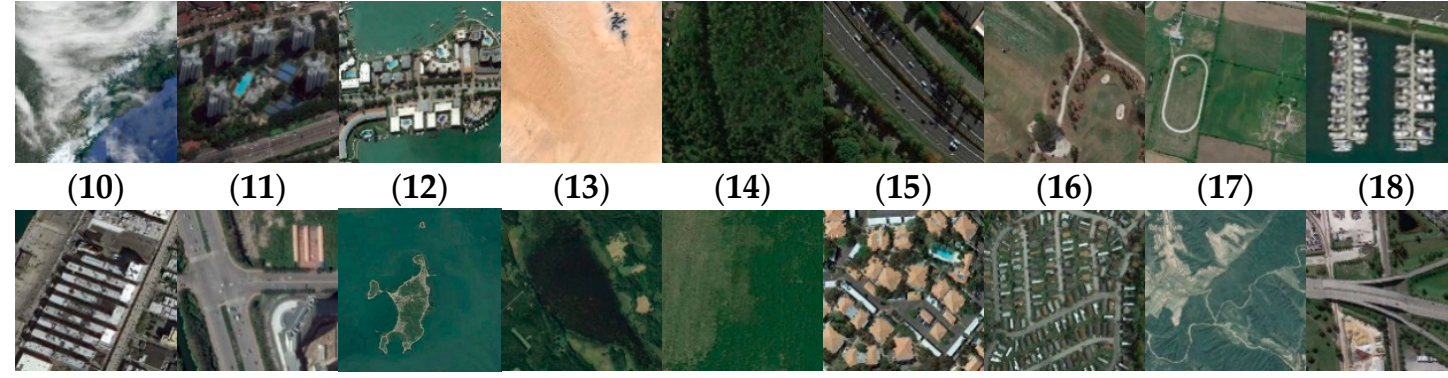

(14)

(15)

(16)

(17)

(18)

(19)

(20)

(21)
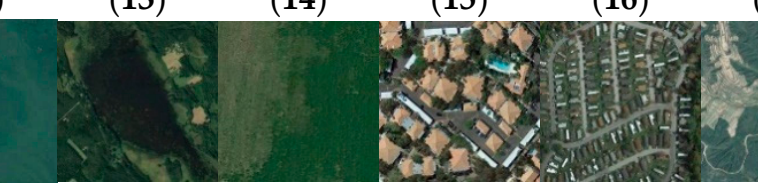

106

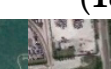

(24)

(25)

(26)

(27)

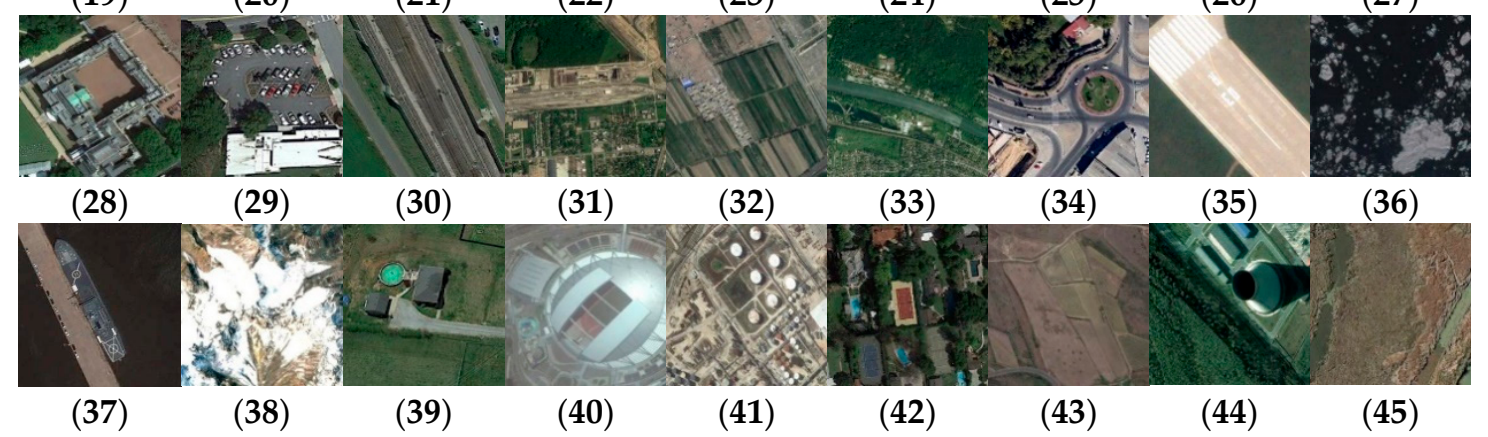

Figure 3. Class representatives of NWPU-RESISC45 dataset: (1) airplane; (2) airport; (3) baseball diamond; (4) basketball court; (5) beach; (6) bridge; (7) chaparral; (8) church; (9) circular farmland; (10) cloud; (11) commercial area; (12) dense residential; (13) desert; (14) forest; (15) freeway; (16) golf course; (17) ground track field; (18) harbor; (19) industrial area; (20) intersection; (21) island; (22) lake; (23) meadow; (24) medium residential; (25) mobile home park; (26) mountain; (27) overpass; (28) palace; (29) parking lot; (30) railway; (31) railway station; (32) rectangular farmland; (33) river; (34) roundabout; (35) runway; (36) sea ice; (37) ship; (38) snow berg; (39) sparse residential; (40) stadium; (41) storage tanks; (42) tennis court; (43) terrace; (44) thermal power station; (45) wetland.

Table 1 summarizes the three datasets. Given the differences between each dataset, experiments on each will help verify the robustness and generalization of our proposed method.

Table 1. Dataset information.

\begin{tabular}{cccccc}
\hline Dataset Name & Number of Classes & Image Size & Resolution/m & Images per Class & Total Images \\
\hline UC Merced & 21 & $256 \times 256$ & 2 & 100 & 2100 \\
AID & 30 & $600 \times 600$ & $0.5 \sim 8$ & $200 \sim 400$ & 10,000 \\
NWPU-RESISC45 & 45 & $256 \times 256$ & $0.2 \sim 30$ & 700 & 31,500 \\
\hline
\end{tabular}

\subsection{Method}

The method in this study integrates the idea of a lightweight CNN MobileNetv2 and a bilinear model for fine-grained visual recognition. MobileNet [66] is a lightweight CNN proposed to apply deep learning on mobile and edge devices. It greatly reduces $\mathrm{CNN}$ parameters and calculations by using depthwise separable convolution. Although the classification accuracy of MobileNet on ImageNet is slightly lower than that of deep CNNs such as ResNet50, it has the unique advantages 
of a smaller size, fewer parameters, fewer calculations and can be used on mobile and embedded devices. MobileNetv2 introduced an inverted residual and linear bottleneck, further compressing parameters and calculations, improving performance. The bilinear model is a widely used method for fine-grained visual recognition. In the bilinear model, two parallel CNNs (which are separated by the last fully connected layers and classification layers) are used as feature extractors to obtain two deep features of the same image. The two features are then in a bilinear pooling instead of a connection, summation, or maximum pooling. Bilinear pooling is an efficient feature fusion strategy. Along with its concise form and gradient calculation method, the bilinear model can also be trained end-to-end and has excellent classification performance in fine-grained visual recognition. In the following sections, we introduce the depthwise separable convolution, linear bottleneck, inverse residual, bilinear model, and the network architecture.

\subsubsection{Depthwise Separable Convolution}

The core idea of depthwise separable convolution (Figure 4) is to divide the traditional standard convolution operation into two steps: depthwise convolution and pointwise convolution. Assuming that the size of the input feature maps is $D_{K} \times D_{K} \times M$, using $N$ convolution kernels of size $D_{K} \times D_{K} \times M$ to perform the convolution operation on the input feature maps, $N$ feature maps of size $D_{R} \times D_{R}$ can be directly obtained, where $D_{R}$ is the width and height of the input feature maps, $M$ is the number of channels of the input feature maps, $D_{K}$ is the width and height of the convolution kernels, $N$ is the number of convolution kernels, and $D_{R}$ is the width and height of the output feature maps. When using depthwise separable convolution to operate a convolution on feature maps of size $D_{R} \times D_{R} \times M$, firstly $M$ convolution kernels of size $D_{K} \times D_{K} \times 1$ are used to convolve with each channel of the feature maps separately. The size of the output feature maps is $D_{R} \times D_{R} \times M$. Depthwise convolution only changes the width and height of the original feature maps but does not change the number of channels. To increase the channels of the feature maps, pointwise convolution can be used after depthwise convolution. In the process of pointwise convolution, $N$ convolution kernels of size $1 \times 1 \times M$ are used to operate convolution on the feature maps to obtain $N$ feature maps of size $D_{R} \times D_{R}$. Finally, the size of the feature maps generated by standard convolution and by depthwise separable convolution are the same, but the number of parameters and calculations has changed. The standard convolution calculation is $D_{K} \times D_{K} \times M \times N \times D_{F} \times D_{F}$, while the depthwise separable convolution calculation is $D_{K} \times D_{K} \times M \times D_{F} \times D_{F}+M \times N \times D_{F} \times D_{F}$. In a CNN, when the size of the convolution kernels is $3 \times 3$, the depthwise separable convolution calculation is $\sim 1 / 9$ of the standard convolution calculation. In addition, in order to further reduce the network parameters, MobileNet introduces two parameters: channel multiplier and resolution multiplier. The channel multiplier, $\alpha$, is used to proportionally expand or reduce the number of feature channels; the resolution multiplier, $\rho$, is used to proportionally enlarge or reduce the size of the feature maps. The calculation of the depthwise separable convolution after reducing the channel number and size of feature maps is $D_{K} \times D_{K} \times \alpha M \times \rho D_{F} \times \rho D_{F}+\alpha M \times \alpha N \times \rho D_{F} \times \rho D_{F}$. 

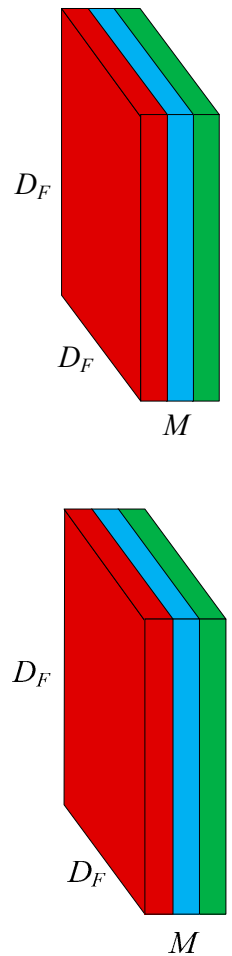

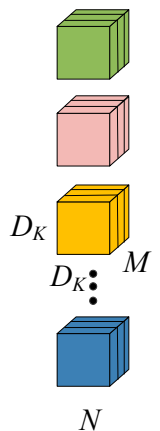

(a)
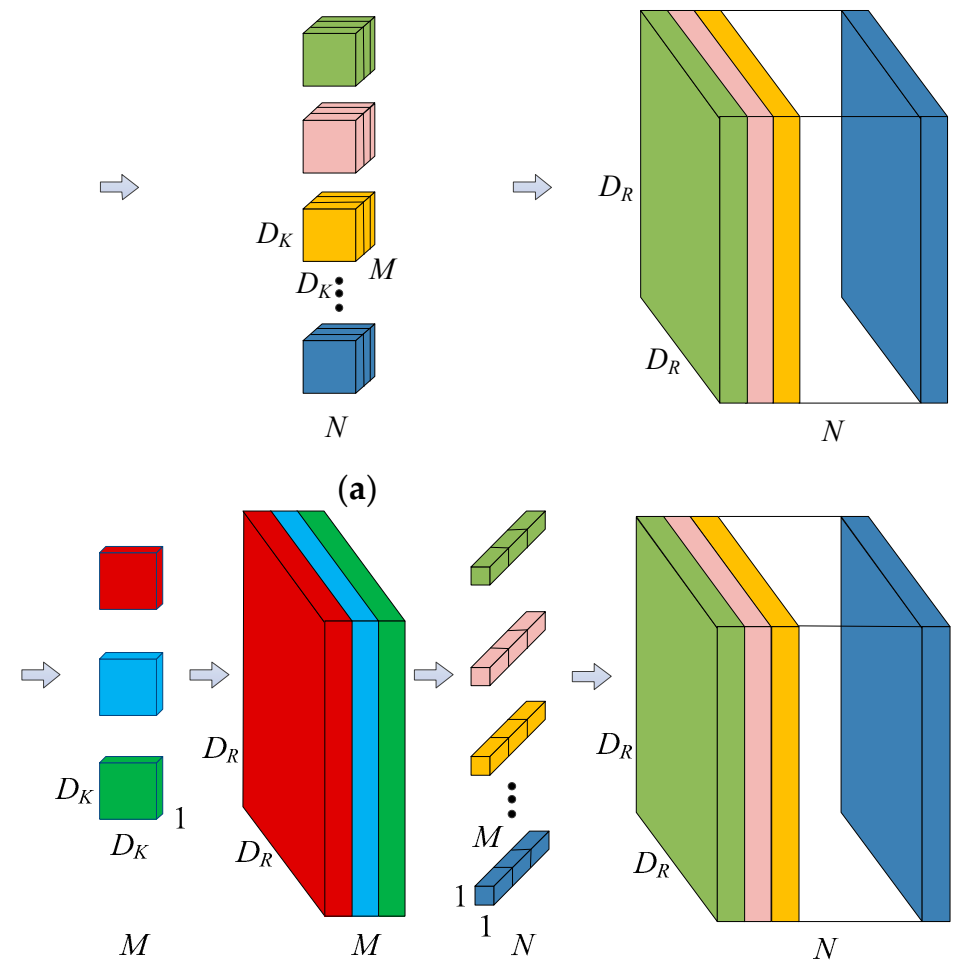

(b)

Figure 4. The difference between (a) standard convolution and (b) depthwise separable convolution. $D_{K}$ is the width and height of the convolution kernels, $D_{R}$ is the width and height of the output feature maps, $M$ is the channel number of the input feature maps and $N$ is the channel number of the output feature maps.

\subsubsection{Linear Bottleneck}

The introduction of the linear bottleneck is to solve the information loss caused by using activation functions such as ReLu (rectified linear unit) in CNNs. The activation function in a CNN generally performs a non-linear transformation on the feature maps of the input. The non-linearity allows the neural network to approximate any arbitrary non-linear function and enhances the network's ability to express information. The ReLu activation function outputs are zero if the input is negative; for positive inputs, the output is dependent on a linear transformation. The ReLu function, therefore, increases the sparsity of the network (outputs of zero are ignored) and reduces the interdependence between parameters, thus reducing the possibility of model overfitting. However, the ReLu function will also cause large losses of information for features with small channels, as the process of feature dimension reduction is also a process of feature compression. The essence of the linear bottleneck, therefore, is that after the pointwise convolutional layer and the batch normalization layer, the feature maps are directly passed to the next convolutional layer without using a non-linear activation function (Figure 5). 


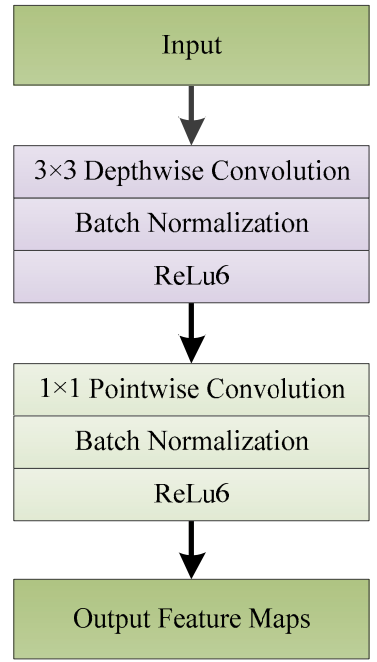

(a)

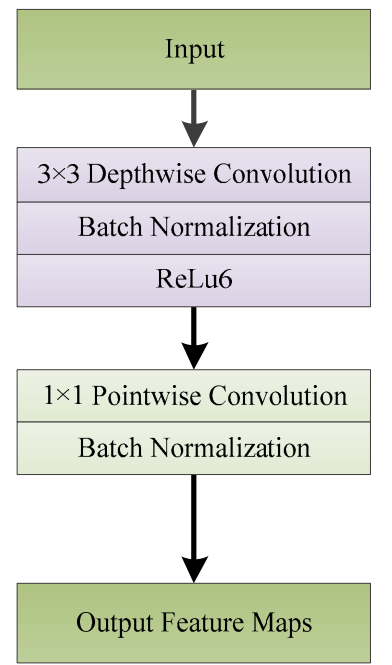

(b)

Figure 5. The basic structure of MobileNet: (a) unit in MobileNetv1 [66]; (b) unit in MobileNetv2 [65].

\subsubsection{Inverted Residual Block}

MobileNetv2 uses the feature shortcut connection idea in the ResNet structure to fuse feature maps between different convolutional layers (Figure 6). When ResNet performs shortcut feature connection, it first uses pointwise convolution to compress the channel number of the input feature maps (usually to 0.25 times the original number). Feature maps after compression are passed to a standard convolution module where the channel number of the feature maps is in constant. The number of channels is restored to the original number using another pointwise convolution. Finally, the feature maps are added to the input feature maps. The inverted residual adopted in MobileNetv2 is the opposite: a complete inverted residual structure first performs a pointwise convolution to expand the number of feature channels to $m$ times the original number ( $m$ is an integer greater than 1 ; in MobileNetv2 the value of $m$ is 6), and then performs depthwise and pointwise convolution. In the second pointwise convolution, the feature map channels are expanded to the original number and then the obtained feature maps are added to the original feature maps. Similarly, the ReLu activation function is no longer used after the second pointwise convolution. The design of the inverted residual structure not only has good memory efficiency, but also improves network performance. 


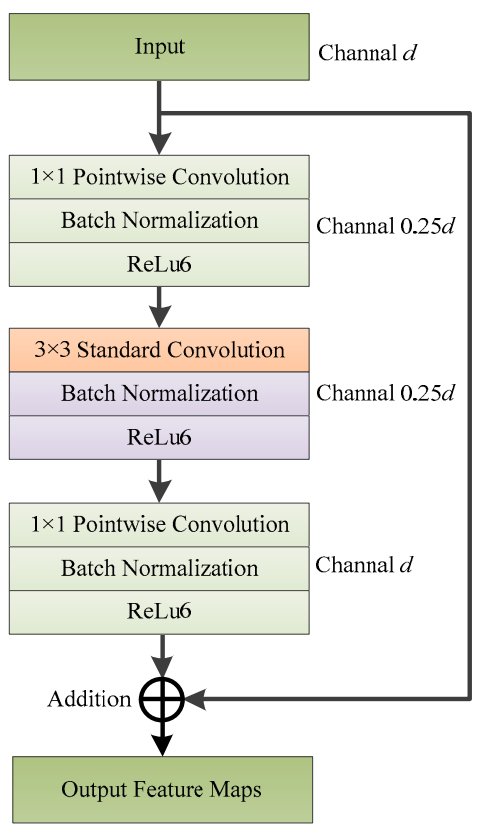

(a)

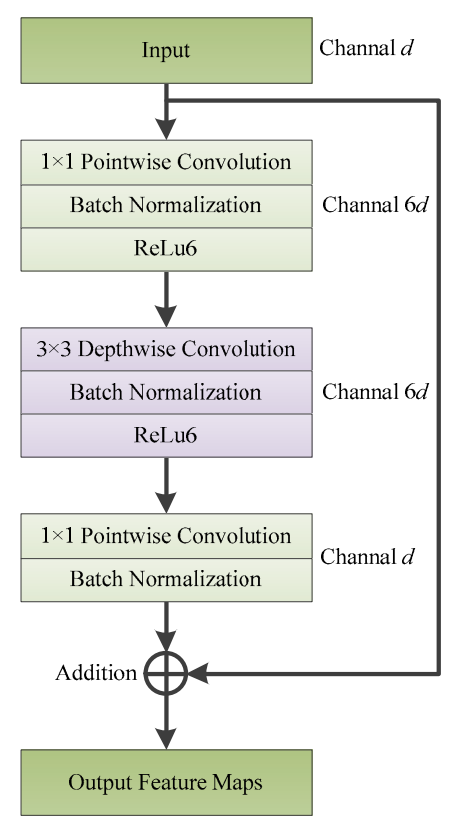

(b)

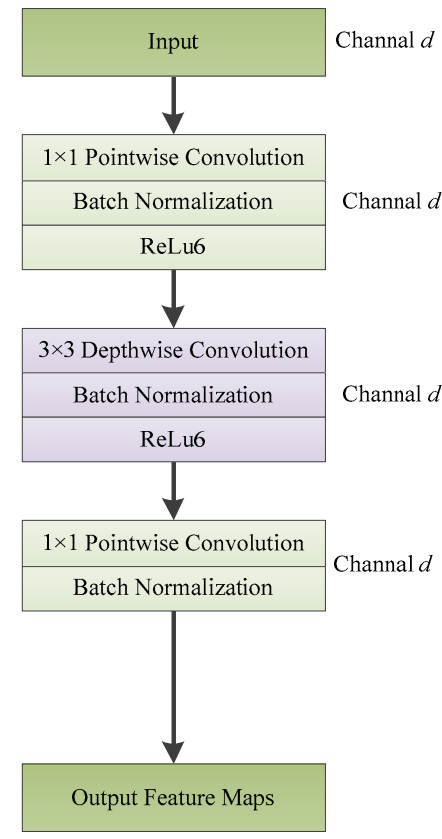

(c)

Figure 6. Blocks in a convolutional neural network (CNN): (a) standard residual block; (b) inverted residual block in MobileNetv2 when stride is 1; (c) linear block in MobileNetv2 when stride is 2.

\subsubsection{Bilinear Model}

Lin et al. [63] first proposed a bilinear CNN model (B-CNN) in fine-grained visual recognition tasks producing excellent performance. The core idea is to use two parallel CNNs to extract features from the same image and then merge the two features using bilinear pooling to obtain a new feature vector (Figure 7). A standard bilinear model $\mathcal{B}$ consists of four components: $\mathcal{B}=\left(f_{\mathrm{A}}, f_{\mathrm{B}}, \mathcal{P}, \mathcal{C}\right)$, where $f_{\mathrm{A}}$ and $f_{\mathrm{B}}$ are two feature extraction functions based on CNNs, which are used to extract features of the same image, $\mathcal{P}$ is a pooling function, and $C$ is a classification function. When two CNNs extract features from the same image $I$ and perform bilinear pooling in position $l$, the outer product operation is used, and the calculation process is as follows:

$$
\begin{gathered}
b\left(l, \boldsymbol{I}, f_{A}, f_{B}\right)=f_{A}^{T}(l, \boldsymbol{I}) f_{B}^{T}(l, \boldsymbol{I}), \\
\xi(\boldsymbol{I})=\sum_{l} b\left(l, \boldsymbol{I}, f_{A}, f_{B}\right), \\
\boldsymbol{x}=\operatorname{vec}(\xi(\boldsymbol{I})), \\
y=\operatorname{sign}(x) \sqrt{|x|}, \\
\boldsymbol{z}=y /\|y\|_{2} .
\end{gathered}
$$

when the size of output feature maps of the input image was $D_{W} \times D_{H} \times C$, feature maps of size $D_{W} \times D_{H} \times C^{2}$ could be obtained through an outer product operation. Each feature map was then summed and pooled globally to obtain a bilinear feature $x$ of size $C^{2}$; a square root operation (Equation (4)) and a normalization operation (Equation (5)) on the bilinear feature were then carried out to obtain a bilinear vector. Finally, logistic regression or a support vector machine (SVM) was used for classification with the bilinear vector. The bilinear model is not only simple in form and procedure but also enables end-to-end training and testing. It also has excellent performance in fine-grained classification tasks. 


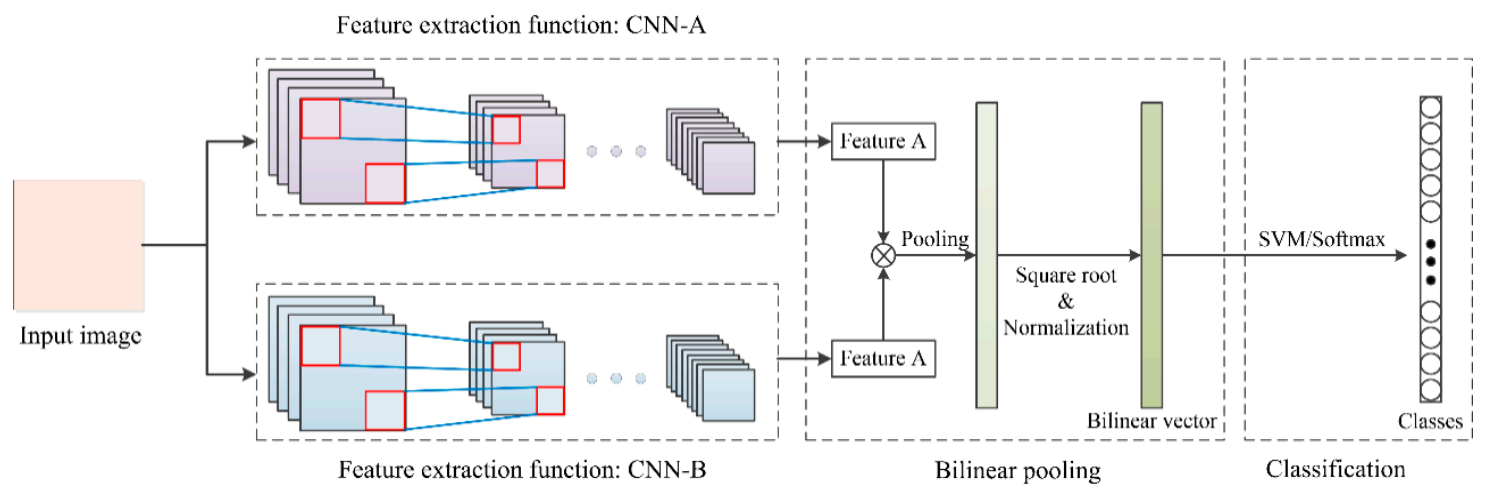

Figure 7. Pipeline of the bilinear-CNN model proposed by Lin et al. [63].

\subsection{Proposed Architecture}

The integration of the structure of MobileNetv2 and the method of feature fusion in the bilinear model, the network structure of BiMobileNet, is shown in Figure 8. The network includes main three parts: feature extraction, bilinear pooling of features to obtain a bilinear vector, and classification of bilinear features.

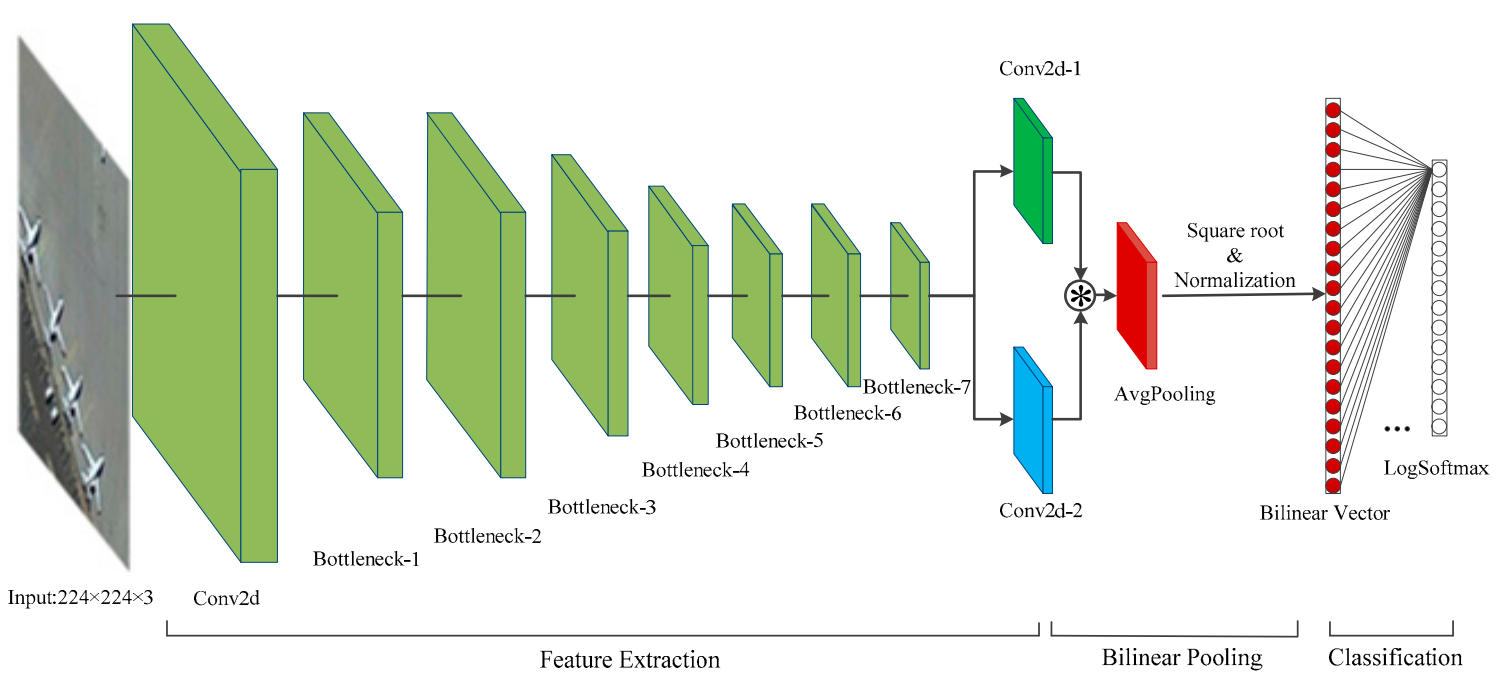

Figure 8. Architecture of BiMobileNet. See text for description.

The backbone network of the feature extraction layer utilizes MobileNetv2 but does not use all layers. Instead, we removed the last three layers: one convolutional layer, one average pooling layer, and one classification layer. This is because the size of feature maps of the last convolutional layer is $7 \times 7 \times 1280$. This still contained too many parameters; we wanted as few as possible. The structure information of BiMobileNetv2 is shown in Table 2. The feature extraction layer contains a convolutional layer and seven bottlenecks. Every bottleneck consisted of a linear or inverted residual block. For example, when an image with size $224 \times 224 \times 3$ passes through the feature extraction layer, feature maps with size of $7 \times 7 \times 320$ are obtained. In BiMobileNet we did not use two convolutional neural networks to extract features. Instead the feature maps extracted from the same network were shared to reduce the parameters and calculations in the model. 
Table 2. BiMobileNet details.

\begin{tabular}{|c|c|c|c|}
\hline Layer Name & Operation & Input Size & Output Size \\
\hline Conv2d & $\begin{array}{c}\text { Conv2d, kernel size }=(3 \times 3), \\
\text { stride }=2\end{array}$ & $224 \times 224 \times 3$ & $112 \times 112 \times 32$ \\
\hline Bottleneck-1 & Linear block, $m=1$, stride $=1$ & $112 \times 112 \times 32$ & $112 \times 112 \times 16$ \\
\hline \multirow{2}{*}{ Bottleneck-2 } & \multirow{2}{*}{$\begin{array}{c}\text { Linear block, } m=6, \text { stride }=2 \\
\text { Inverted residual block, } m=6, \\
\text { stride }=1\end{array}$} & $112 \times 112 \times 16$ & $56 \times 56 \times 24$ \\
\hline & & $56 \times 56 \times 24$ & $56 \times 56 \times 24$ \\
\hline \multirow{3}{*}{ Bottleneck-3 } & \multirow{3}{*}{$\begin{array}{l}\text { Linear block, } m=6, \text { stride }=2 \\
\text { Inverted residual block, } m=6, \\
\text { stride }=1 \\
\text { Inverted residual block, } m=6, \\
\text { stride }=1\end{array}$} & $56 \times 56 \times 24$ & $28 \times 28 \times 32$ \\
\hline & & $28 \times 28 \times 32$ & $28 \times 28 \times 32$ \\
\hline & & $28 \times 28 \times 32$ & $28 \times 28 \times 32$ \\
\hline \multirow{4}{*}{ Bottleneck-4 } & \multirow{4}{*}{$\begin{array}{c}\text { Linear block, } m=6, \text { stride }=1 \\
\text { Inverted residual block, } m=6, \\
\text { stride }=1 \\
\text { Inverted residual block, } m=6, \\
\text { stride }=1 \\
\text { Inverted residual block, } m=6, \\
\text { stride }=1\end{array}$} & $28 \times 28 \times 32$ & $28 \times 28 \times 64$ \\
\hline & & $28 \times 28 \times 64$ & $28 \times 28 \times 64$ \\
\hline & & $28 \times 28 \times 64$ & $28 \times 28 \times 64$ \\
\hline & & $28 \times 28 \times 64$ & $28 \times 28 \times 64$ \\
\hline \multirow{3}{*}{ Bottleneck-5 } & \multirow{3}{*}{$\begin{array}{c}\text { Linear block, } m=6, \text { stride }=2 \\
\text { Inverted residual block, } m=6, \\
\text { stride }=1 \\
\text { Inverted residual block, } m=6, \\
\text { stride }=1\end{array}$} & $28 \times 28 \times 64$ & $14 \times 14 \times 96$ \\
\hline & & $14 \times 14 \times 96$ & $14 \times 14 \times 96$ \\
\hline & & $14 \times 14 \times 96$ & $14 \times 14 \times 96$ \\
\hline \multirow{3}{*}{ Bottleneck-6 } & \multirow{3}{*}{$\begin{array}{c}\text { Linear block, } m=6, \text { stride }=2 \\
\text { Inverted residual block, } m=6, \\
\text { stride }=1 \\
\text { Inverted residual block, } m=6, \\
\text { stride }=1\end{array}$} & $14 \times 14 \times 96$ & $7 \times 7 \times 160$ \\
\hline & & $7 \times 7 \times 160$ & $7 \times 7 \times 160$ \\
\hline & & $7 \times 7 \times 160$ & $7 \times 7 \times 160$ \\
\hline Bottleneck-7 & Linear block, $m=6$, stride $=1$ & $7 \times 7 \times 160$ & $7 \times 7 \times 320$ \\
\hline \multirow{3}{*}{ Bilinear Pooling } & $\begin{array}{l}\text { Conv2d-1, } \begin{array}{l}\text { kernel size }=(k \times k), \\
\text { stride }=1\end{array}\end{array}$ & $7 \times 7 \times 320$ & $7 \times 7 \times 1024$ \\
\hline & $\begin{array}{l}\text { Conv2d-2, } \text { kernel size }=(k \times k), \\
\text { stride }=1\end{array}$ & $7 \times 7 \times 320$ & $7 \times 7 \times 1024$ \\
\hline & AvgPooling kernel size $=(7 \times 7)$ & $7 \times 7 \times 1024$ & $1 \times 1 \times 1024$ \\
\hline Classification & Fully Connected & $1 \times 1 \times 1024$ & class number \\
\hline
\end{tabular}

Before bilinear fusion of the obtained features, inspired by the hierarchical bilinear model proposed in [64], we used two feature transformation layers on the extracted features, thereby transforming the same feature into two different, but similar, features. The feature transformation layer is essentially a convolutional layer, with a size of 1024 convolution kernels of size $k \times k$ (here $k$ is 3 ). After the feature maps with size $7 \times 7 \times 320$ pass through the feature transformation layer, two kinds of feature maps with size $7 \times 7 \times 1024$ are generated. The original bilinear model used the outer product operation when performing bilinear pooling operations on two different features. This expanded the dimensions of the obtained feature maps by $C$ times ( $C$ is the channel number of feature maps); the dimensions of the bilinear feature vector were also expanded $C$ times. When the channel number of the two features is 1024, million-dimensional feature vectors were generated. Not only is the model prone to overfitting, but also the training and implementation time is significant. Using the Hadamard product, instead of the outer product, in the bilinear model keeps the dimensions of the original feature maps unchanged. The Hadamard product is the multiplication of the elements at the corresponding positions in the two matrices of the same order and does not change the dimension of the matrices. In the bilinear 
fusion in BiMobileNet, two feature maps with size $7 \times 7 \times 1024$ are processed by the Hadamard product to generate bilinear features with size $7 \times 7 \times 1024$, keeping the feature dimensions unchanged. The average pooling operation is performed on the bilinear features with the size $7 \times 7 \times 1024$; a bilinear feature vector with a size of $1 \times 1 \times 1024$ is obtained. The classification layer is a fully connected layer. Its input is a bilinear feature vector with size of $1 \times 1 \times 1024$ and the probability of each category is the output.

\subsection{Experimental Setup}

\subsubsection{Implementation Details}

Before the experiment, each dataset was divided into training and test sets. In order for comparisons with other methods, we adopted different training ratios for the experiments. The training and test sets were chosen randomly from the original dataset. Every experiment was performed five times. The mean and standard deviation of the five results were calculated. By rotating the training images $90^{\circ}, 180^{\circ}$, and $270^{\circ}$ clockwise, horizontal flip and vertical flip, we expanded the training data six-fold. This augmentation helped to generalize the CNN models.

We utilized the open source deep learning framework PyTorch to build BiMobileNet. BiMobileNet can be trained end-to-end, using stochastic gradient descent to update its parameters. As BiMobileNet shares part of the MobileNetv2 network structure, before training, we used MobileNetv2's pre-trained weight on ImageNet to initialize parameters. The hyperparameter settings in BiMobileNet were as follows: the initial learning rate of the feature extraction layers was 0.01 , while the initial learning rate of the bilinear pooling layer and the classification layer was 0.1 . Every 10 epochs, the learning rate was reduced by 0.5 times. The momentum and weight decay were 0.9 and 0.0005 , respectively. The training batch size was 32, and the number of training epochs was 100 . We use the well-trained model whose training loss is stable to predict the test set. All experiments were performed on a device with Intel Core i7-6900K CPU 64-GB RAM and GeForce GTX1080Ti GPU 11-GB RAM.

\subsubsection{Evaluation Protocol}

BiMobileNet was comprehensively evaluated from aspects of classification accuracy and model complexity. The accuracy was expressed by two criteria: confusion matrix and overall accuracy. In the confusion matrix, each row represents the true class and each column represents its predicted category. The elements on the diagonal in the confusion matrix represent the classification accuracy of one class and the elements on the non-diagonal $\mathrm{CM}_{i j}$ represent the probability that the images from class $i$ th are mistakenly recognized as class $j$ th. The overall accuracy is defined as the number of correctly predicted images divided by the total number of predicted images.

The model complexity includes time complexity and space complexity. Time complexity indicates the number of model operations, determines the training and prediction time of the model, and is represented by floating point operations (FLOPs). The higher the time complexity, the slower the model speed. For the model with high time complexity, model training and prediction time is long, which is less favorable for practical training and application. Space complexity indicates the space size of the model, which can be represented by the model size and the total number of model parameters. A model with considerable parameters needs a large amount of data to train and it is very easy to over fit.

\section{Results}

\subsection{Classification of the UC Merced Dataset}

The training ratios were set at 20\%,50\%, and $80 \%$. Table 3 shows the classification performance comparison of our architecture compared to the state-of-the-art methods on the UC Merced dataset. By analyzing the overall accuracies obtained by state-of-the-art methods under different training 
ratios, we find that on the UC Merced dataset, when using $80 \%$ of the data for training, the overall accuracy of other state-of-the-art methods are very close to $99.00 \%$. When the training ratio is $80 \%$, the classification accuracy becomes saturated, and it is difficult to improve it further. Using $80 \%$ of data for training, BiMobileNet nearly achieves the highest overall accuracy ( $99.03 \%$ compared to $99.05 \%)$; using $50 \%$ of data for training, BiMobileNet achieves the highest overall accuracy of $98.45 \%$ - this is better than many methods which use a training ratio of $80 \%$. When training with $20 \%$ data, the classification accuracy of BiMobileNet reaches $96.41 \%$. On one hand, BiMobileNet achieves outstanding performance when the training ratio is $50 \%$ (similar result to $80 \%$ training ratio); however, it also demonstrates that the classification accuracy is saturated when the training ratio is $80 \%$, and it is difficult to further improve accuracy. We must consider model complexity and other issues with a small training sample.

Table 3. Overall accuracy of the state-of-the-art method on UC Merced dataset. The highest accuracy for each ratio is bolded.

\begin{tabular}{|c|c|c|c|c|}
\hline \multirow{2}{*}{ Method } & \multirow{2}{*}{ Published Year } & \multicolumn{3}{|c|}{ Training Ratio } \\
\hline & & $20 \%$ & $50 \%$ & $80 \%$ \\
\hline BOVW [4] & 2010 & & & 76.81 \\
\hline VLAT [67] & 2014 & & & 94.30 \\
\hline MS-CLBP+FV [34] & 2016 & & $88.76 \pm 0.79$ & $93.00 \pm 1.20$ \\
\hline TEX-NET-FL (ResNet) [51] & 2017 & & $96.91 \pm 0.36$ & $97.72 \pm 0.54$ \\
\hline salM³ LBP-CLM [54] & 2017 & & $91.21 \pm 0.75$ & $95.75 \pm 0.80$ \\
\hline VGG-VD-16 [2] & 2017 & & $94.14 \pm 0.69$ & $95.21 \pm 1.20$ \\
\hline CNN-ELM [68] & 2017 & & & $95.62 \pm 0.32$ \\
\hline Two-Stream Fusion [69] & 2018 & & & $98.02 \pm 1.03$ \\
\hline D-CNN (VGG16) [47] & 2018 & & & $98.93 \pm 0.10$ \\
\hline RTN (VGG16) [42] & 2018 & & & 98.96 \\
\hline DCF (VGG-VD16) [70] & 2018 & & $95.42 \pm 0.71$ & $97.10 \pm 0.85$ \\
\hline GCFs+LOFs (VGG16) [9] & 2018 & & $97.37 \pm 0.44$ & $99.00 \pm 0.35$ \\
\hline SAL-TS-Net (GoogLeNet) [8] & 2018 & & $97.97 \pm 0.56$ & $98.90 \pm 0.95$ \\
\hline Siamese ResNet50 [71] & 2019 & 76.50 & 90.95 & 94.29 \\
\hline SF-CNN (VGGNet) [44] & 2019 & & & $99.05 \pm 0.27$ \\
\hline VGG16-DF [43] & 2019 & & & 5298.97 \\
\hline MRBF [72] & 2019 & & & $94.19 \pm 0.15$ \\
\hline DDRL-AM (ResNet18) [41] & 2019 & & & $99.05 \pm 0.08$ \\
\hline WSPM-CRC (ResNet152) [73] & 2019 & & & 97.95 \\
\hline CTFCNN [52] & 2019 & & & $98.44 \pm 0.58$ \\
\hline CapsNet (Inception-v3) [74] & 2019 & & $97.59 \pm 0.16$ & $99.05 \pm 0.24$ \\
\hline BiMobileNet (MobileNetv2) & 2020 & $96.41 \pm 0.57$ & $98.45 \pm 0.27$ & $99.03 \pm 0.28$ \\
\hline
\end{tabular}

Most of the state-of-the-art methods in Table 3 adopt deep CNNs (such as VGG16, ResNet50, etc.). Generally, deep CNNs with many layers and parameters have a large calculation component (the parameters and calculation are discussed in detail in Section 3.3), and the training of these networks requires a large amount of data. Therefore, it is difficult to train these networks with a small amount of training data, and overfitting often occurs. In addition, for a classification task, it is unreasonable and unrealistic to use $80 \%$ of the data for training. This is because annotation is a time-consuming job, and manually labeling $80 \%$ of the data is unfeasible. For practical applications, it is necessary to reduce manual annotation as much as possible and improve classification accuracy and efficiency with as little data as possible. However, BiMobileNet can still achieve an accuracy of $96.41 \%$ when only $20 \%$ of the data is used for training. In order to further verify BiMobileNet performance with little training data, more training ratios $(5 \%, 10 \%, 15 \%, 20 \%$ and $25 \%$ ) were used (Table 4$)$. When the training ratio was $5 \%$ (e.g., five images randomly selected from each category for training and 95 images are predicted), BiMobileNet achieved an amazing overall accuracy of $86.74 \%$, which was far higher than the results of fine-tuning VGG16, ResNet50 and MobileNetv2 directly. That means when faced with a new larger dataset, we can only label a very small portion of the data for training and then predict the remaining 
data, which can save a lot of labor and time. When the training ratio was $20 \%$, BiMobileNet achieved an overall accuracy of $96.41 \%$ while the method proposed by Chaib [57] reached $92.96 \%$. For the other lower training ratios, BiMobileNet also achieved excellent performance. This demonstrates that the method in this study is not prone to overfitting when using little training data, and has a large accuracy advantage over the deep CNNs in scene classification task using the UC Merced dataset.

Table 4. Overall accuracy of BiMobileNet under different training ratios. The highest accuracy for each ratio is bolded.

\begin{tabular}{cccccc}
\hline \multirow{2}{*}{ Method } & \multicolumn{5}{c}{ Training Ratio } \\
\cline { 2 - 6 } & $\mathbf{5 \%}$ & $\mathbf{1 0 \%}$ & $\mathbf{1 5 \%}$ & $\mathbf{2 0 \%}$ & $\mathbf{2 5 \%}$ \\
\hline Fine-tuning VGG16 & $39.53 \pm 2.23$ & $53.12 \pm 1.15$ & $59.83 \pm 2.45$ & $64.68 \pm 2.70$ & $69.51 \pm 0.65$ \\
Fine-tuning ResNet50 & $39.01 \pm 1.62$ & $51.35 \pm 1.25$ & $57.40 \pm 0.96$ & $64.82 \pm 0.64$ & $71.06 \pm 0.72$ \\
Fine-tuning MobileNetv2 & $38.64 \pm 1.45$ & $52.85 \pm 0.85$ & $60.90 \pm 1.26$ & $67.86 \pm 1.12$ & $72.48 \pm 0.40$ \\
BiMobileNet & $\mathbf{8 6 . 7 4} \pm \mathbf{1 . 6 3}$ & $\mathbf{9 3 . 7 8} \pm \mathbf{0 . 7 5}$ & $\mathbf{9 3 . 9 0} \pm \mathbf{0 . 2 5}$ & $\mathbf{9 6 . 4 1} \pm \mathbf{0 . 5 7}$ & $\mathbf{9 7 . 0 2} \pm \mathbf{0 . 5 5}$ \\
\hline
\end{tabular}

Figures 9-12 show the confusion matrices for training ratios of 5\%, 10\%, 20\%, and 50\%, respectively, on the UC Merced dataset. When the training ratio is $5 \%, 11$ of the 21 scene categories achieve a classification accuracy greater than $92 \%$, and only four categories: buildings (0.73), dense residential (0.33), intersection (0.76), and river (0.57), are lower than $80 \%$. Many dense residential images (which have the lowest accuracy) are misidentified as medium residential and mobile home park, as the three types are very similar. With little training data (5 images), it is difficult to distinguish the three categories effectively for a CNN. When the training ratio is $10 \%, 16$ of the 21 scene categories achieve a classification accuracy of greater than $92 \%$; dense residential has the lowest accuracy (67\%) but twice as high compared to the $5 \%$ training ratio. When the training ratio is $20 \%, 16$ of the 21 scene categories achieve a classification accuracy of greater than $95 \%$. When the training ratio is $50 \%, 18$ of the 21 scene categories achieve a classification accuracy of greater than $98 \%$; the three lower accuracy categories (building, dense residential, and medium residential) still achieve $92 \%$. These three categories have poorer accuracy, because buildings are the main image component. Images were originally annotated based on building density, but this is a subjective and perceptual judgment, with no quantitative standard explaining the lower model accuracy.

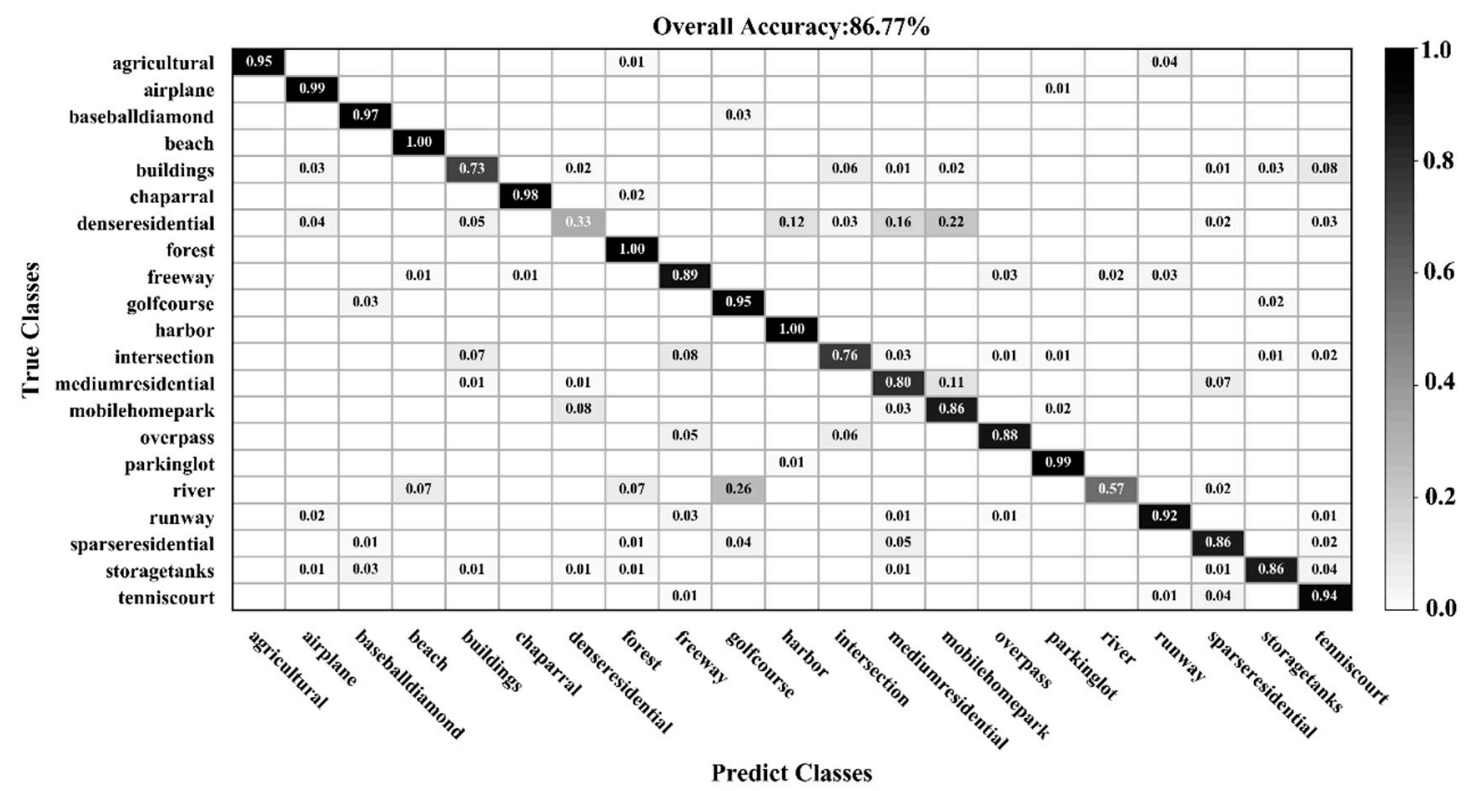

Figure 9. Confusion matrix using a training ratio of $5 \%$ on the UC Merced dataset. 


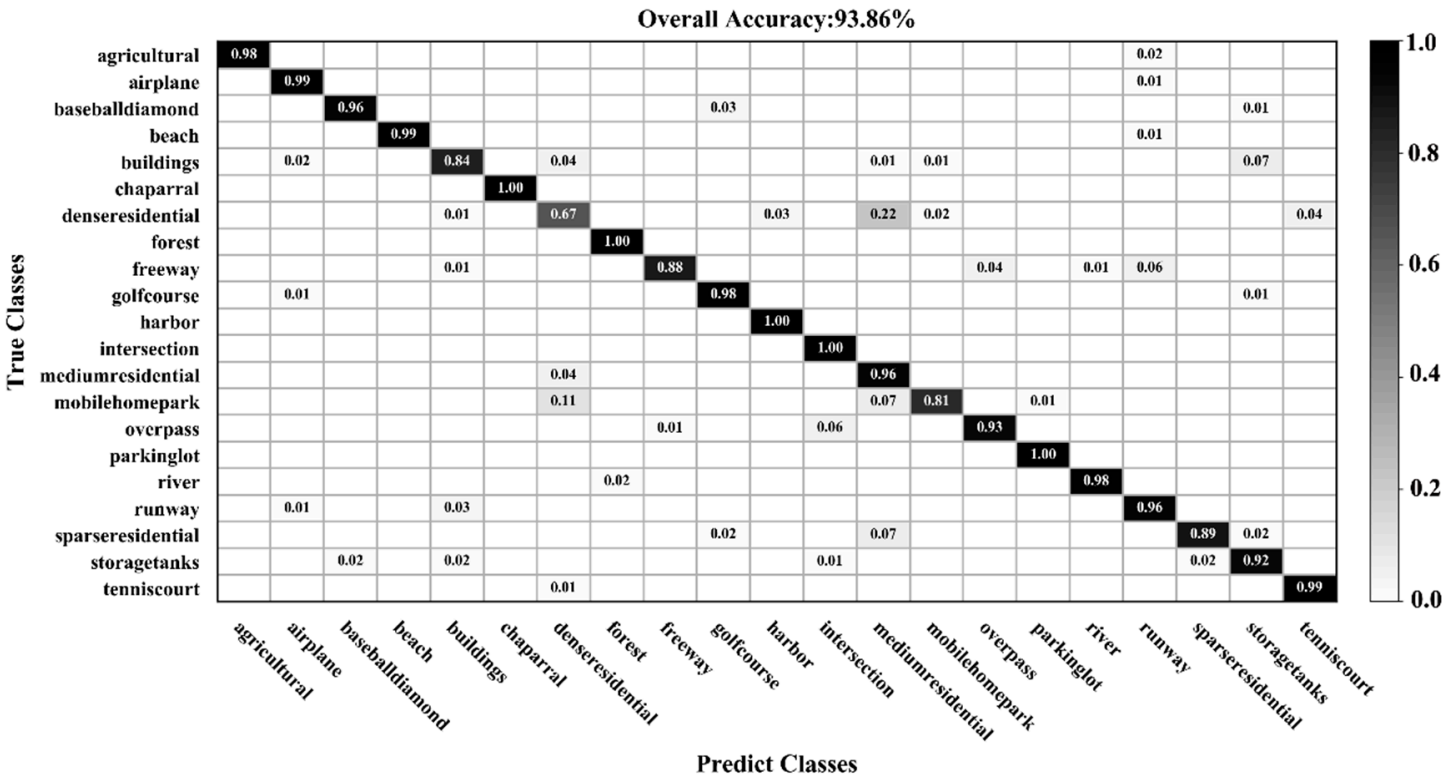

Figure 10. Confusion matrix using a training ratio of $10 \%$ on the UC Merced dataset.

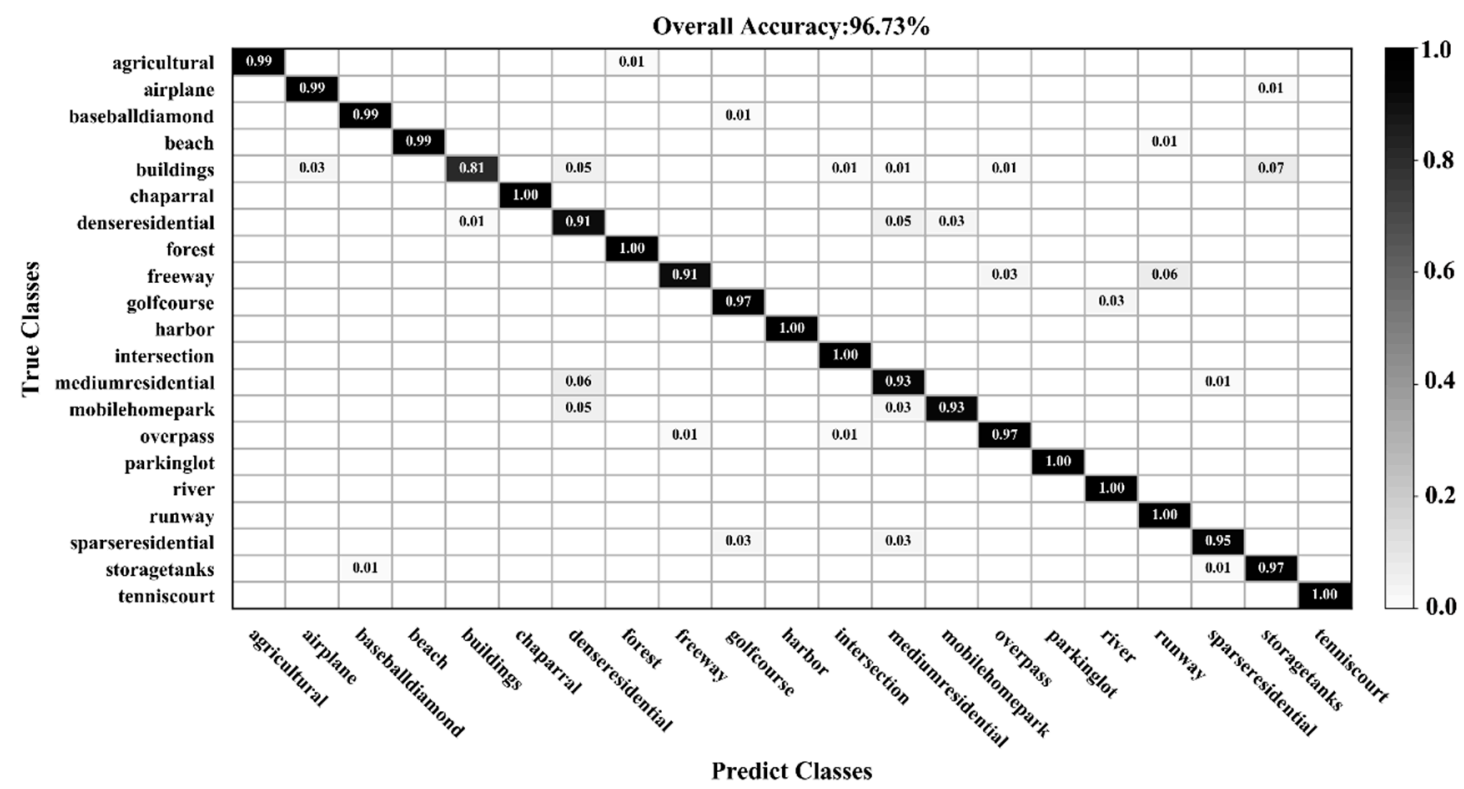

Figure 11. Confusion matrix using a training ratio of $20 \%$ on the UC Merced dataset. 


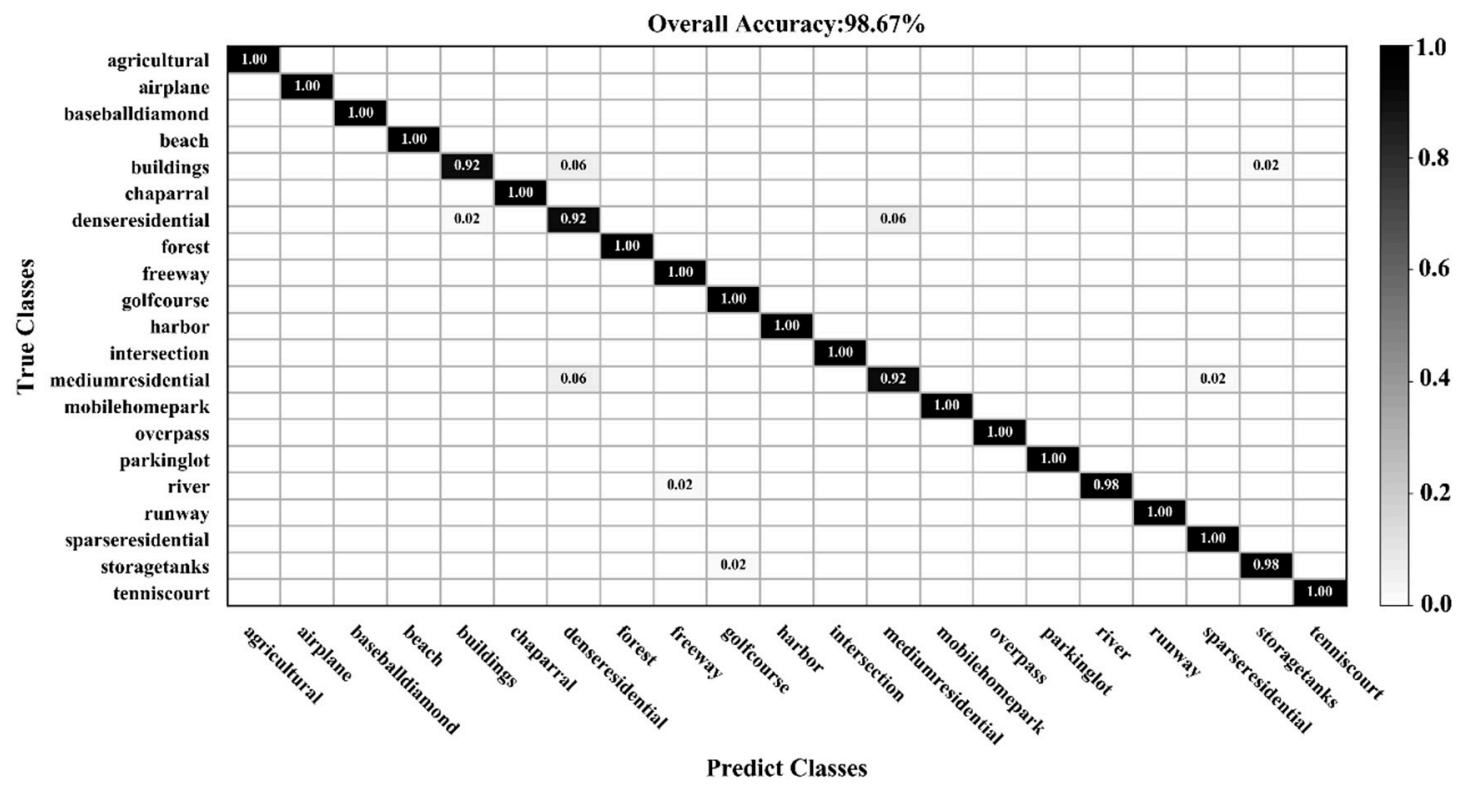

Figure 12. Confusion matrix using a training ratio of $50 \%$ on the UC Merced dataset.

\subsection{Classification of the AID Dataset}

When using the AID dataset, training ratios were set at $10 \%, 20 \%$, and $50 \%$. Table 5 compares the accuracy of state-of-the-art methods with our approach. Using a training ratio of $50 \%$, the overall classification accuracy of BiMobileNet is $96.87 \%$, which is higher than most other methods. When the training ratio is $20 \%$, the overall accuracy is $94.83 \%$, which is $\sim 1 \%$ higher than all other methods. When the training ratio is $10 \%$, the overall accuracy is $92.77 \%$. BiMobileNet produces a similar accuracy to D-CNN [47], GCFs+LOFs [9] and SF-CNN [44] when using a training ratio of $50 \%$ but performs $\sim 4.0 \%, \sim 2.5 \%$, and $\sim 1.2 \%$, respectively, higher when the training ratio is $20 \%$. The D-CNN, GCFs+LOFs, and SF-CNN networks all adopt VGG16, the parameters and calculations of which are much larger than MobileNetv2 utilized in BiMobileNet.

Comparing Tables 3 and 5, it is noted that the classification accuracy of different methods on the AID dataset is generally lower than the UC Merced dataset for a given training ratio. This is because the AID dataset has more categories, and the data is more diverse, rendering classification more challenging. Figures 13-15 show the confusion matrices when the training ratios of the AID dataset are set to $10 \%, 20 \%$, and $50 \%$, respectively. When the training ratios are $10 \%$ and $20 \%, 22$ and 27 categories (out of 30), respectively, have a classification accuracy greater than $91 \%$. When the training ratio is $50 \%$, the accuracy of most categories is greater than $98 \%$. As training data increases, the classification accuracy of most categories improves significantly. However, the accuracy of the resort class is $76 \%$ (10\% training ratio), $72 \%(20 \%)$, and $83 \%(50 \%)$-lower than all other classes. Some images from the resort category are mistaken for park. This is mainly because park and resort have a similar object (buildings, vegetation) distribution. In addition, school and commercial, and center and square have similar features. Consequently, the school and resort classes have relatively low classification accuracies compared with other categories when the training ratio of the AID dataset is set to $50 \%$. 
Table 5. Overall accuracy of the state-of-the-art methods on AID dataset. The highest accuracy for each ratio is bolded.

\begin{tabular}{|c|c|c|c|c|}
\hline \multirow{2}{*}{ Method } & \multirow{2}{*}{ Published Year } & \multicolumn{3}{|c|}{ Training Ratio } \\
\hline & & $10 \%$ & $20 \%$ & $50 \%$ \\
\hline TEX-Net-LF (ResNet) [51] & 2017 & & $93.81 \pm 0.12$ & $95.73 \pm 0.16$ \\
\hline salM³ LBP-CLM [54] & 2017 & & $86.92 \pm 0.35$ & $89.76 \pm 0.45$ \\
\hline VGG-VD-16 [2] & 2017 & & $86.59 \pm 0.29$ & $89.64 \pm 0.36$ \\
\hline DCA (VGGNet) [57] & 2017 & & & $91.86 \pm 0.28$ \\
\hline RTN (VGG16) [42] & 2018 & & 92.44 & \\
\hline D-CNN (VGG16) [47] & 2018 & & $90.82 \pm 0.16$ & $96.89 \pm 0.10$ \\
\hline GCFs+LOFs (VGG16) [9] & 2018 & & $92.48 \pm 0.38$ & $96.85 \pm 0.23$ \\
\hline SAL-TS-Net (GoogleNet) [8] & 2018 & & $94.09 \pm 0.34$ & $95.99 \pm 0.35$ \\
\hline MRBF [72] & 2019 & & & $87.26 \pm 0.42$ \\
\hline SF-CNN (VGGNet) [44] & 2019 & & $93.60 \pm 0.12$ & $96.66 \pm 0.11$ \\
\hline CTFCNN [52] & 2019 & & & $94.91 \pm 0.24$ \\
\hline WSPM-CRC (ResNet152) [73] & 2019 & & & 95.11 \\
\hline BiMobileNet (MobileNetv2) & 2020 & $92.77 \pm 0.49$ & $94.83 \pm 0.24$ & $96.87 \pm 0.23$ \\
\hline
\end{tabular}

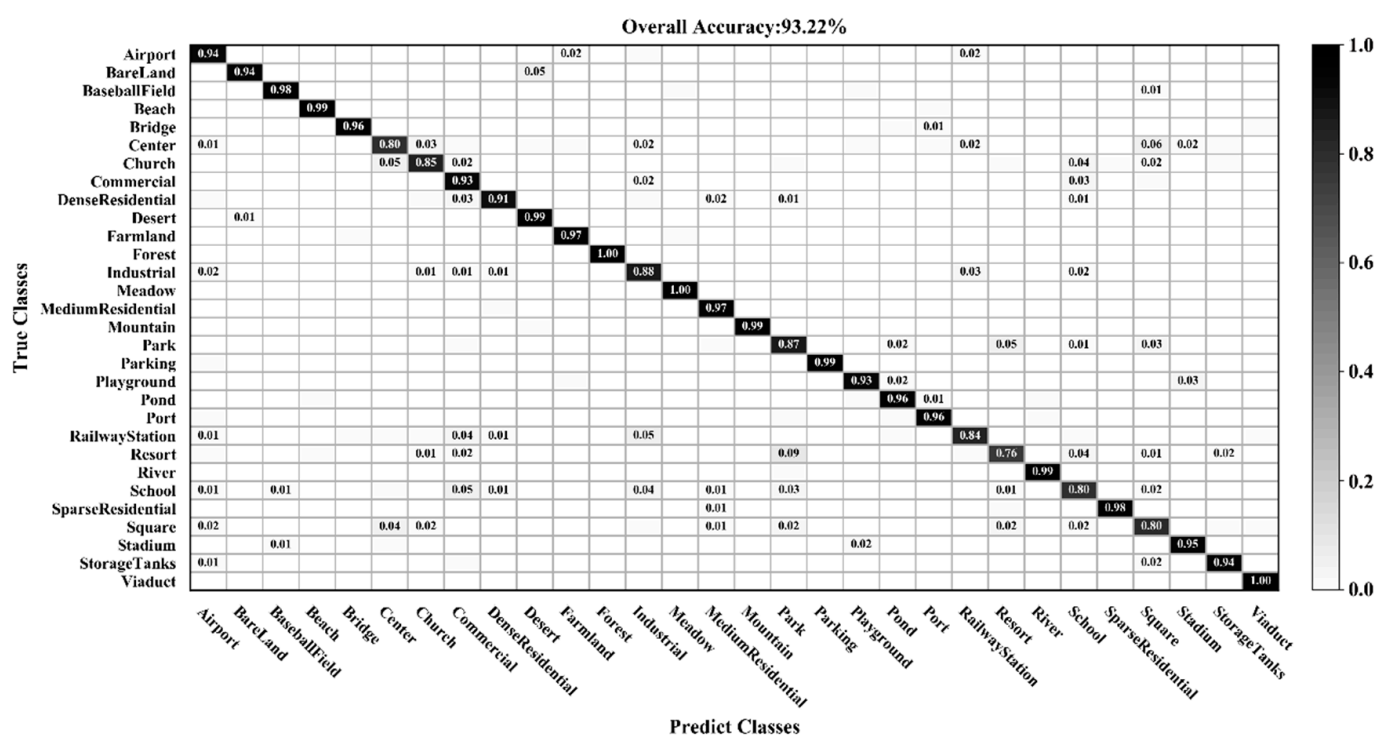

Figure 13. Confusion matrix using a training ratio of $10 \%$ on the AID dataset.

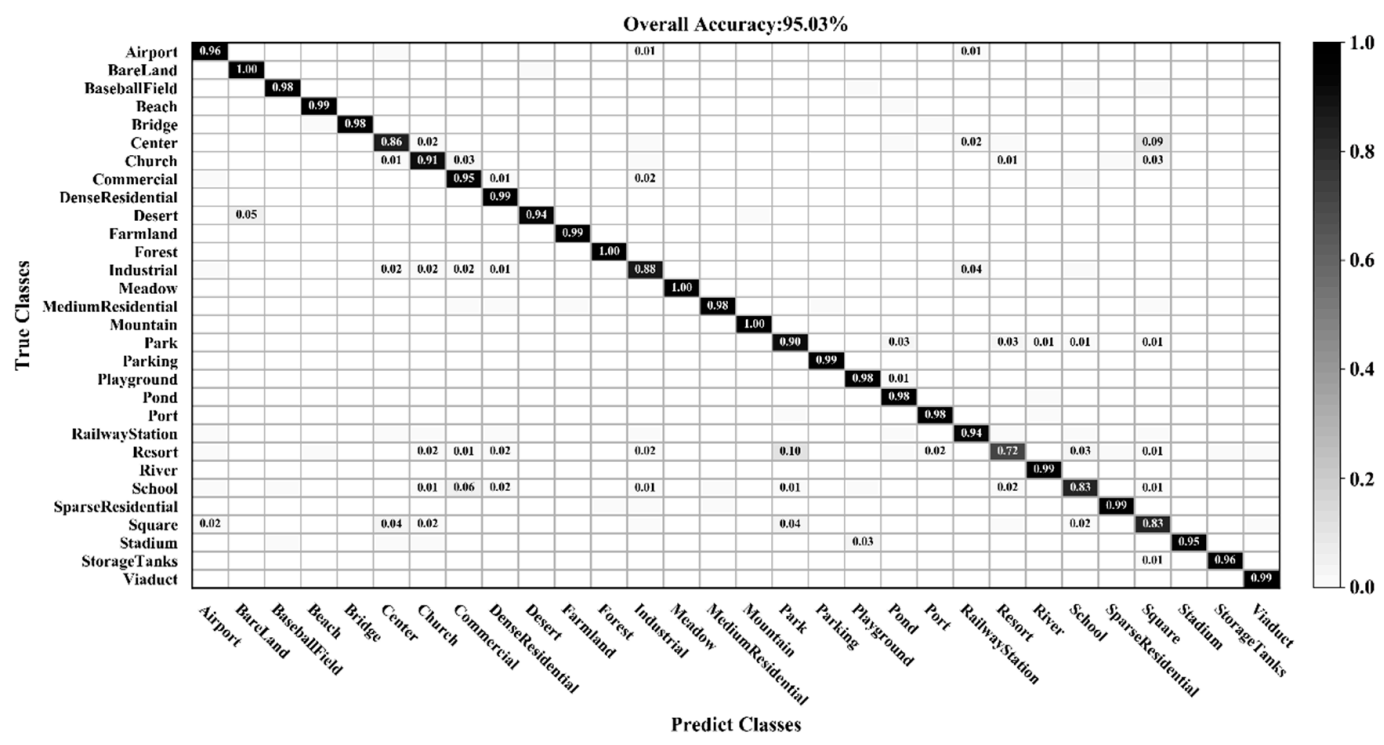

Figure 14. Confusion matrix using a training ratio of $20 \%$ on the AID dataset. 


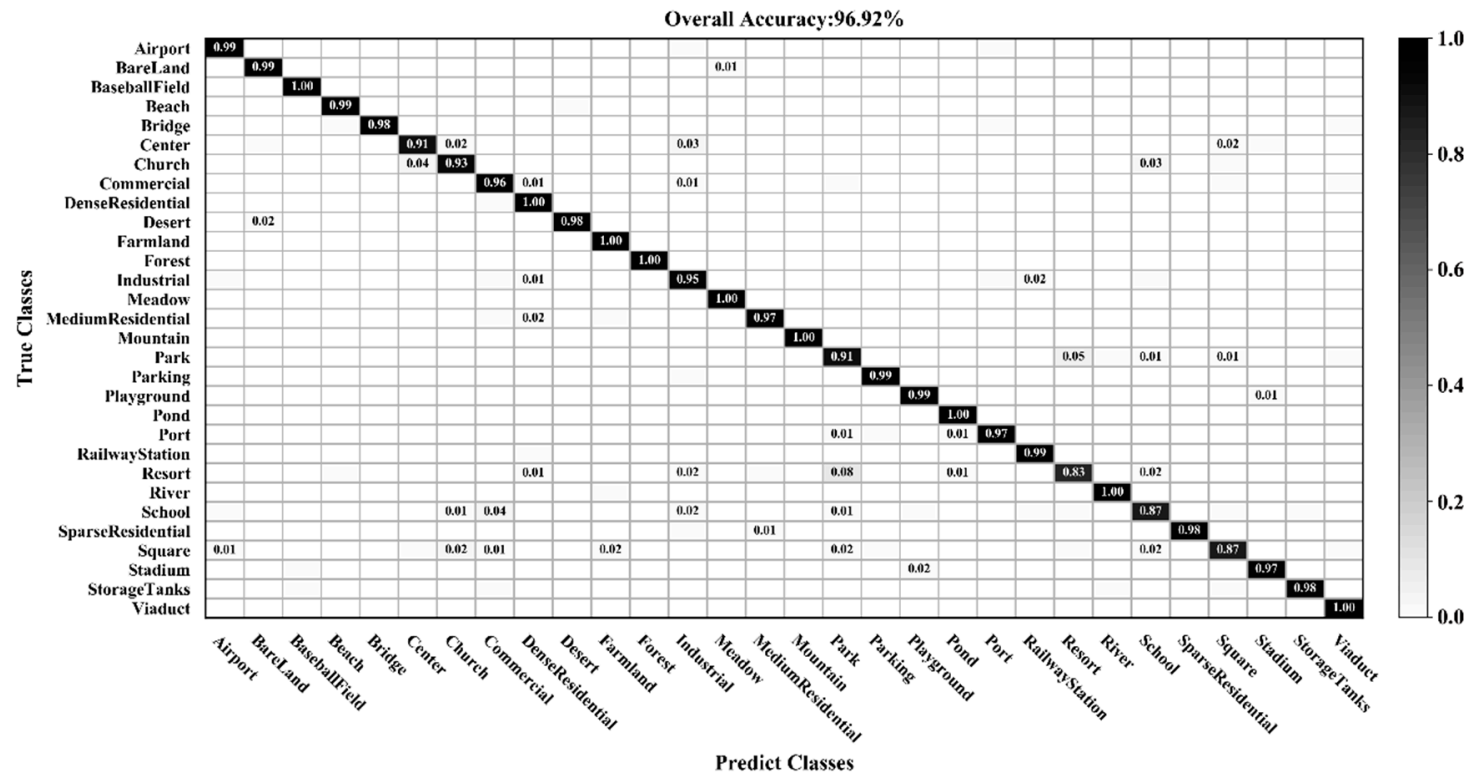

Figure 15. Confusion matrix using a training ratio of $50 \%$ on the AID dataset.

\subsection{Classification of the NWPU-RESISC45 Dataset}

For the NWPU-RESISC45 dataset, training data ratios were set to $10 \%$ and $20 \%$. The classification accuracies of state-of-the-art methods and BiMobileNet are shown in Table 6. The NWPU-RESISC45 dataset has more categories and images than the other two datasets. The overall accuracy of BiMobileNet is $92.06 \%$ and $94.08 \%$ when the training ratios are $10 \%$ and $20 \%$, respectively; this is higher than all but one other methods. When the training ratio is $10 \%$, BiMobileNet accuracy is $2.1 \%, 1.0 \%$ and $0.3 \%$ higher than SF-CNN [44], GLANet [46] and DML [49], respectively, and is similar to DDRL-AM [41]. SF-CNN, GLANet, and DML adopt deep CNN VGGNet; DDRL-AM adopts deep CNN ResNet18. The parameters and calculation of these two networks are significantly larger than MobieNetv2 used in BiMobileNet. In addition, when the training ratio is $20 \%$, BiMobileNet accuracy is $\sim 1.5 \%, \sim 0.6 \%$, $\sim 0.6 \%$, and $\sim 1.6 \%$ better than SF-CNN, GLANet, DML and DDRL-AM, respectively.

Figures 16 and 17 show the confusion matrixes obtained by BiMobileNet using training ratios of $10 \%$ and $20 \%$, respectively, on the NWPU-RESISC 45 dataset. When the training ratio is $10 \%$, the classification accuracy of 35 categories is greater than $90 \%$. When the training ratio is $20 \%$, the classification accuracy of 41 categories is greater than $90 \%$; for GLANet, 38 categories are greater than $90 \%$. As the training ratio increases, classification accuracy of most categories significantly improves. Although the accuracy of the church (72\% with $10 \%$ ratio, $75 \%$ with $20 \%$ ratio) and palace $(68 \%, 78 \%)$ categories improve, the accuracy is still significantly lower than other categories. This is because the two categories have similar architectural styles and layouts that can easily be misclassified. 
Table 6. Overall accuracy of the state-of-the-art methods on NWPU-RESISC45 dataset. The highest accuracy for each ratio is bolded.

\begin{tabular}{cccc}
\hline Method & \multirow{2}{*}{ Published Year } & \multicolumn{2}{c}{ Training Ratio } \\
\cline { 3 - 4 } & & $\mathbf{1 0} \%$ & $\mathbf{2 0 \%}$ \\
\hline Fine-tune VGG16 [3] & 2017 & $87.15 \pm 0.45$ & $90.36 \pm 0.18$ \\
D-CNN (VGG16) [47] & 2018 & $89.22 \pm 0.50$ & $91.89 \pm 0.22$ \\
IOR4 (VGG16) [40] & 2018 & $87.83 \pm 0.16$ & $91.30 \pm 0.17$ \\
RTN (VGG16) [42] & 2018 & 89.90 & 92.71 \\
DCF (VGG-VD16) [70] & 2018 & $87.14 \pm 0.19$ & $89.56 \pm 0.25$ \\
DDRL-AM (ResNet18) [41] & 2018 & $\mathbf{9 2 . 1 7} \pm \mathbf{0 . 0 8}$ & $92.46 \pm 0.09$ \\
SAL-TS-Net (GoogLeNet) [8] & 2018 & $85.02 \pm 0.20$ & $87.01 \pm 0.19$ \\
Triplet Network [7] & 2018 & & $92.33 \pm 0.50$ \\
VGG16-DF [43] & 2019 & 89.66 & \\
Siamese ResNet50 [71] & 2019 & & 92.28 \\
SF-CNN (VGG16) [44] & 2019 & $89.89 \pm 0.16$ & $92.55 \pm 0.14$ \\
GLANet [46] & 2019 & $91.03 \pm 0.18$ & $93.45 \pm 0.17$ \\
CapsNet (Inception-v3) [74] & 2019 & $89.03 \pm 0.21$ & $92.60 \pm 0.11$ \\
DML (VGG16) [49] & 2019 & $91.73 \pm 0.21$ & $93.47 \pm 0.30$ \\
BiMobileNet (MobileNetv2) & 2020 & $92.06 \pm 0.14$ & $\mathbf{9 4 . 0 8} \pm \mathbf{0 . 1 1}$ \\
\hline
\end{tabular}

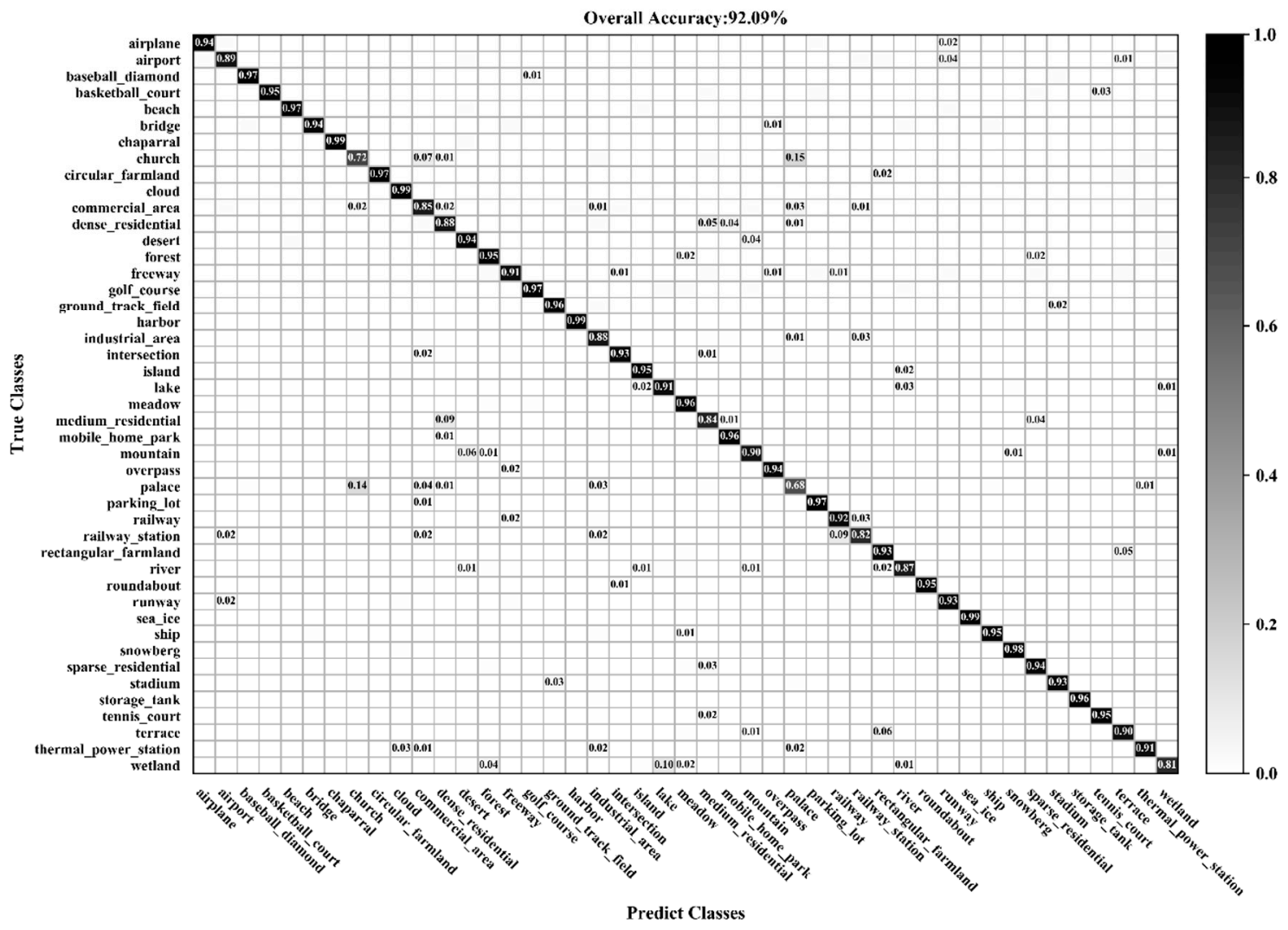

Figure 16. Confusion matrix using a training ratio of $10 \%$ on the NWPU-RESISC45 dataset. 


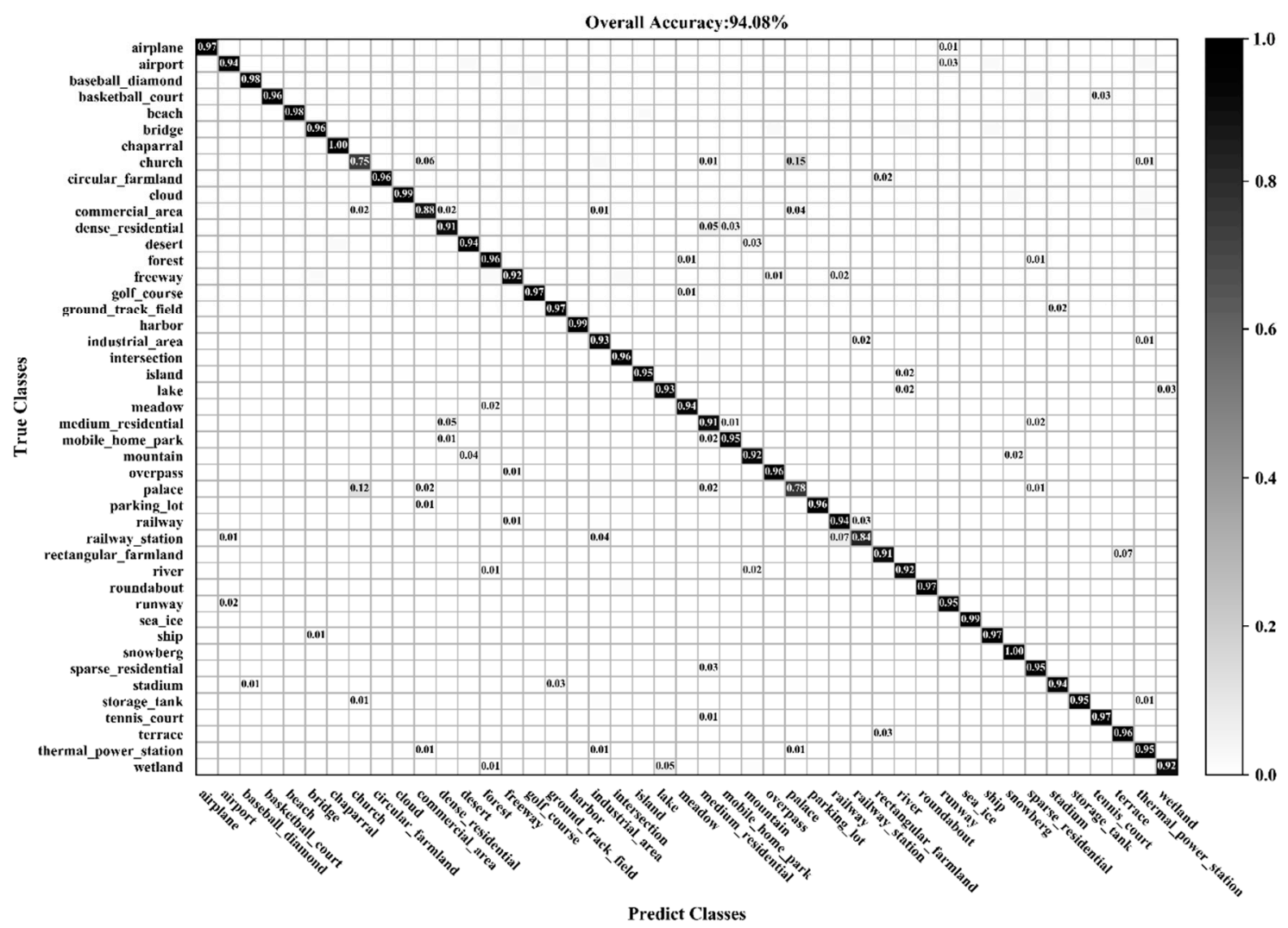

Figure 17. Confusion matrix using a training ratio of $20 \%$ on the NWPU-RESISC45 dataset.

\section{Discussion}

Currently, most remote sensing image scene classification methods only take classification accuracy into account, and seldom consider parameters, calculations and other issues. From our experimental results on three datasets (Tables 3, 5 and 6), the majority of methods use deep CNNs such as VGG16 [56], GoogLeNet [58], and ResNet [59]. Although deep CNNs show strong generalization for image classification and object detection, they have clear disadvantages such as too many parameters and significant computation time for training and predictive processes. For example, the model size of VGG16 exceeds $512 \mathrm{MB}$, and the number of parameters exceeds 134 million. These directly affect the training and prediction time. More importantly, deep CNNs are easy to over fit with little sample data. Moreover, such deep CNNs can only be trained and implemented on hardware devices with high computational performance; this is not always conducive to practical application and deployment. Although CNNs such as GoogLeNet and ResNet have significantly reduced parameters and computational cost compared with VGG16, their parameters and computation are still significant and not suitable for mobile or other edge devices. In this case, the model with fewer parameters and less computation is more suitable for practical application, especially for real-time classification and object detection. In other words, the deployment of efficient and lightweight CNNs don't not require high-end equipment and can also achieve better performance. For the task of remote sensing image classification, we may need to deploy the model on UAV (unmanned aerial vehicle), small satellite and other devices in the future to achieve real-time classification. Therefore, the model we designed not only needs to have outstanding performance, but also needs to focus on faster speed and less computation. That's the advantage of our approach using MobileNet.

Comparison of different state-of-the-art methods on overall accuracy, parameters, calculation and model size are shown in Table 7. Many algorithms were improved on VGG16 or ResNet such as SF-CNN [44], DML [49], etc. As can be seen from the Table 7, VGG16 has the most parameters and calculations. SF-CNN replaced the last two fully connected layers with convolutional layers and adopted global mean pooling in the classification layer in VGG16. SF-CNN reduced the number of 
parameters but did not fundamentally reduce the calculations compared to VGG16. DML [49] did not change the structure of VGG16 but adopted mean center loss. Although it improved the accuracy of the original VGG16 in remote sensing image scene classification, it did not change the structure of the VGG16, and the number of parameters and calculations did not decrease. SAL-TS-Net [8] merged the features from two GoogLeNet networks in parallel. Compared with VGG16, the parameters and computational cost are reduced, but the precision is lower than directly fine-tuning VGG16. The BiMobileNet we designed has the least parameter and computation but achieved higher accuracies. Different channel reduction factors $\lambda$ are set in BiMobileNet to further reduce model parameters and calculations. When $\lambda$ is 0.75 and $k$ is 1 , the overall accuracy of BiMobileNet is higher than that of most methods. The number of parameters is approximately $1 / 11,1 / 85$, and $1 / 6$ that of SF-CNN, DML and SAL-TS-Net, respectively. The calculation is approximately $1 / 65,1 / 65$ and $1 / 6$ that of SF-CNN, DML, and SAL-TS-Net, respectively. Compared with other state-of-the-art methods, obviously BiMobileNet not only has outstanding performance, but also significantly reduces parameters and calculational cost.

Table 7. Accuracy and complexity of state-of-the-art methods on NWPU-RESISC45 dataset. The highest accuracy and least calculation are bolded.

\begin{tabular}{|c|c|c|c|c|c|c|}
\hline \multirow{2}{*}{ Methods } & \multirow{2}{*}{$\lambda$} & \multicolumn{2}{|c|}{ Overall Accuracy } & \multirow{2}{*}{$\begin{array}{l}\text { Parameters Numbers } \\
\text { (Million) }\end{array}$} & \multirow{2}{*}{ GFLOPs $^{1}$} & \multirow{2}{*}{$\begin{array}{c}\text { Model Size } \\
\text { MB }\end{array}$} \\
\hline & & $10 \%$ & $20 \%$ & & & \\
\hline Fine-tuning VGG16 [3] & 1 & $87.15 \pm 0.45$ & $90.36 \pm 0.18$ & $\sim 134.44$ & $\sim 15.60$ & $\sim 512.87$ \\
\hline SF-CNN (VGG16) [44] & / & $89.89 \pm 0.16$ & $92.55 \pm 0.14$ & $\sim 17.28$ & $\sim 15.49$ & $\sim 65.93$ \\
\hline DML (VGG16) [49] & / & $91.73 \pm 0.21$ & $93.47 \pm 0.30$ & $\sim 134.44$ & $\sim 15.60$ & $\sim 512.87$ \\
\hline $\begin{array}{c}\text { SAL-TS-Net } \\
\text { (GoogLeNet) [8] }\end{array}$ & I & $85.02 \pm 0.20$ & $87.01 \pm 0.19$ & $\sim 10.07$ & $\sim 1.51$ & $\sim 38.41$ \\
\hline \multirow{3}{*}{$\begin{array}{l}\text { BiMobileNet } \\
\quad(k=3)\end{array}$} & 0.50 & $90.26 \pm 0.23$ & $92.77 \pm 0.14$ & 3.47 & 0.17 & 13.27 \\
\hline & 0.75 & $91.47 \pm 0.16$ & $93.64 \pm 0.12$ & 5.52 & 0.33 & 21.05 \\
\hline & 1.00 & $92.06 \pm 0.14$ & $94.08 \pm 0.11$ & 7.76 & 0.45 & 29.59 \\
\hline \multirow{3}{*}{$\begin{array}{l}\text { BiMobileNet } \\
\quad(k=1)\end{array}$} & 0.50 & $90.06 \pm 0.11$ & $92.74 \pm 0.11$ & 0.86 & 0.12 & 3.27 \\
\hline & 0.75 & $91.23 \pm 0.09$ & $93.67 \pm 0.05$ & 1.59 & 0.24 & 6.05 \\
\hline & 1.00 & $91.89 \pm 0.19$ & $93.92 \pm 0.11$ & 2.52 & 0.34 & 9.59 \\
\hline
\end{tabular}

${ }^{1}$ GFLOPs $=10^{9}$ floating-point operations; $k$ is the kernel size in bilinear pooling layer.

\section{Conclusions}

This study introduces the idea of a bilinear model in fine-grained image classification into the remote sensing image scene classification task. Based on MobileNetv2, a highly efficient lightweight convolutional neural network $(\mathrm{CNN})$ for remote sensing image scene classification is proposed - BiMobileNet. MobileNetv2 has the advantages of fewer parameters and a smaller number of calculations, but its remote sensing image classification performance is generally weaker than deep CNNs. MobileNetv2's backbone network is used to extract the features of the images, with the features bilinearly pooled to increase intra-class consistency and inter-class distinction which can significantly improve the accuracy of scene classification and be applied to any remote sensing classification task. By training and testing on three widely used large-scale remote sensing image datasets, both the accuracy and complexity of the model were evaluated with the following conclusions drawn:

1 The accuracy of BiMobileNet in remote sensing image scene classification surpasses most state-of-the-art methods, particularly with little training data.

2 BiMobileNet requires fewer parameters and calculations making training and prediction faster and more efficient.

3 The challenges of remote sensing image scene classification are intra-class inconsistency and inter-class indistinction. The method of using bilinear pooling overcomes some of the difficulties of scene classification providing a simple and efficient method for scene classification. 
In addition, compared with the ImageNet dataset (with 1000 categories), current remote sensing image datasets have far fewer categories (NWPU-RESISC 45 dataset has 45 categories, the AID dataset has 30 categories, and the UC Merced dataset has 21 categories). Image categories are more diverse and complex than this, limiting practical applications. However, the use of the lightweight and efficient CNN described in this study will aid faster and more accurate classification of remote sensing images.

Author Contributions: All the authors made significant contributions to the work. Methodology, D.Y.; Project administration, H.G.; Resources, C.Z. and Y.L.; Software, D.L.; Supervision, Q.X.; Writing—original draft, D.Y.; Writing-review \& editing, Q.X., H.G., C.Z., Y.L. and D.L. All authors have read and agreed to the published version of the manuscript.

Funding: This work was funded by the National Natural Science Foundation of China under Grant 41601507.

Acknowledgments: The authors would also like to express their sincere thanks to the editors and the anonymous reviewers for their valuable comments and contributions.

Conflicts of Interest: The authors declare no conflict of interest.

\section{References}

1. Gómez-Chova, L.; Tuia, D.; Moser, G.; Camps-Valls, G. Multimodal classification of remote sensing images: A review and future directions. Proc. IEEE 2015, 103, 1560-1584. [CrossRef]

2. Xia, G.S.; Hu, J.; Hu, F.; Shi, B.; Bai, X.; Zhong, Y.; Zhang, L.; Lu, X. AID: A benchmark data set for performance evaluation of aerial scene classification. IEEE Trans. Geosci. Remote Sens. 2017, 55, 3965-3981. [CrossRef]

3. Cheng, G.; Han, J.; Lu, X. Remote sensing image scene classification: Benchmark and state of the art. Proc. IEEE 2017, 105, 1865-1883. [CrossRef]

4. Yang, Y.; Newsam, S. Bag-of-visual-words and spatial extensions for land-use classification. In Proceedings of the 18th SIGSPATIAL International Conference on Advances in Geographic Information Systems, San Jose, CA, USA, 2-5 November 2010; pp. 270-279.

5. Gu, Y.; Wang, Y.; Li, Y. A survey on deep learning-driven remote sensing image scene understanding: Scene classification, scene retrieval and scene-guided object detection. Appl. Sci. 2019, 9, 2110. [CrossRef]

6. He, X.; Zou, Z.; Tao, C.; Zhang, J. Combined Saliency with multi-convolutional neural network for high resolution remote sensing scene classification. Acta Geod. Cartogr. Sin. 2016, 45, 1073-1080.

7. Liu, Y.; Huang, C. Scene classification via triplet networks. IEEE J. Sel. Top. Appl. Earth Obs. Remote Sens. 2018, 11, 220-237. [CrossRef]

8. Yu, Y.; Liu, F. Dense connectivity based two-stream deep feature fusion framework for aerial scene classification. Remote Sens. 2018, 10, 1158. [CrossRef]

9. Zeng, D.; Chen, S.; Chen, B.; Li, S. Improving remote sensing scene classification by integrating global-context and local-object features. Remote Sens. 2018, 10, 734. [CrossRef]

10. Swain, M.J.; Ballard, D.H. Color indexing. Int. J. Comput. Vis. 1991, 7, 11-32. [CrossRef]

11. Haralick, R.M.; Shanmugam, K.; Dinstein, I.H. Textural features for image classification. IEEE Trans. Syst. Man Cybern. 1973, 6, 610-621. [CrossRef]

12. Luo, B.; Jiang, S.; Zhang, L. Indexing of remote sensing images with different resolutions by multiple features. IEEE J. Sel. Top. Appl. Earth Obs. Remote Sens. 2013, 6, 1899-1912. [CrossRef]

13. Bhagavathy, S.; Manjunath, B.S. Modeling and detection of geospatial objects using texture motifs. IEEE Trans. Geosci. Remote Sens. 2006, 44, 3706-3715. [CrossRef]

14. Dos Santos, J.A.; Penatti, O.A.B.; da Silva Torres, R. Evaluating the Potential of Texture and Color Descriptors for Remote Sensing Image Retrieval and Classification. In Proceedings of the 5th International Conference on Computer Vision Theory and Applications, Angers, France, 17-21 May 2010; pp. 203-208.

15. Aptoula, E. Remote sensing image retrieval with global morphological texture descriptors. IEEE Trans. Geosci. Remote Sens. 2014, 52, 3023-3034. [CrossRef]

16. Newsam, S.; Wang, L.; Bhagavathy, S.; Manjunath, B.S. Using texture to analyze and manage large collections of remote sensed image and video data. Appl. Opt. 2004, 43, 210-217. [CrossRef]

17. Risojević, V.; Momić, S.; Babić, Z. Gabor descriptors for aerial image classification. In Proceedings of the International Conference on Adaptive and Natural Computing Algorithms, Ljubljana, Slovenia, 14-16 April 2011; pp. 51-60. 
18. Dalal, N.; Triggs, B. Histograms of oriented gradients for human detection. In Proceedings of the International Conference on Computer Vision \& Pattern Recognition (CVPR'05), San Diego, CA, USA, 20-25 June 2005; pp. 886-893.

19. Lowe, D.G. Distinctive image features from scale-invariant keypoints. Int. J. Comput. Vis. 2004, 60, 91-110. [CrossRef]

20. Yang, Y.; Newsam, S. Comparing SIFT descriptors and Gabor texture features for classification of remote sensed imagery. In Proceedings of the 15th IEEE International Conference on Image Processing (ICIP 2008), San Diego, CA, USA, 12-15 October 2008; pp. 1852-1855.

21. Shao, W.; Yang, W.; Xia, G.S.; Liu, G. A hierarchical scheme of multiple feature fusion for high-resolution satellite scene categorization. In Proceedings of the International Conference on Computer Vision Systems, St. Petersburg, Russia, 16-18 July 2013; pp. 324-333.

22. Yang, Y.; Newsam, S. Geographic image retrieval using local invariant features. IEEE Trans. Geosci. Remote Sens. 2013, 51, 818-832. [CrossRef]

23. Sheng, G.; Yang, W.; Xu, T.; Sun, H. High-resolution satellite scene classification using a sparse coding based multiple feature combination. Int. J. Remote Sens. 2012, 33, 2395-2412. [CrossRef]

24. Chen, S.; Tian, Y. Pyramid of spatial relatons for scene-level land use classification. IEEE Trans. Geosci. Remote Sens. 2015, 53, 1947-1957. [CrossRef]

25. Wu, H.; Liu, B.; Su, W.; Zhang, W.; Sun, J. Hierarchical coding vectors for scene level land-use classification. Remote Sens. 2016, 8, 436. [CrossRef]

26. Zhou, L.; Zhou, Z.; Hu, D. Scene classification using a multi-resolution bag-of-features model. Pattern Recognit. 2013, 46, 424-433. [CrossRef]

27. Zhao, L.J.; Tang, P.; Huo, L.Z. Land-use scene classification using a concentric circle-structured multiscale bag-of-visual-words model. IEEE J. Sel. Top. Appl. Earth Obs. Remote Sens. 2014, 7, 4620-4631. [CrossRef]

28. Lazebnik, S.; Schmid, C.; Ponce, J. Beyond bags of features: Spatial pyramid matching for recognizing natural scene categories. In Proceedings of the 2006 IEEE Computer Society Conference on Computer Vision and Pattern Recognition (CVPR'06), New York, NY, USA, 17-22 June 2006; pp. 2169-2178.

29. Yang, Y.; Newsam, S. Spatial pyramid co-occurrence for image classification. In Proceedings of the 2011 IEEE International Conference on Computer Vision, Barcelona, Spain, 6-13 November 2011; pp. 1465-1472.

30. Zhao, B.; Zhong, Y.; Zhang, L. Scene classification via latent Dirichlet allocation using a hybrid generative/discriminative strategy for high spatial resolution remote sensing imagery. Remote Sens. Lett. 2013, 4, 1204-1213. [CrossRef]

31. Văduva, C.; Gavăt, I.; Datcu, M. Latent dirichlet allocation for spatial analysis of satellite images. IEEE Trans. Geosci. Remote Sens. 2012, 51, 2770-2786. [CrossRef]

32. Zhong, Y.; Cui, M.; Zhu, Q.; Zhang, L. Scene classification based on multi-feature probabilistic latent semantic analysis for high spatial resolution remote sensing images. J. Appl. Remote Sens. 2015, 9, 0950640. [CrossRef]

33. Zhao, B.; Zhong, Y.; Zhang, L.; Huang, B. The Fisher kernel coding framework for high spatial resolution scene classification. Remote Sens. 2016, 8, 157. [CrossRef]

34. Huang, L.; Chen, C.; Li, W.; Du, Q. Remote sensing image scene classification using multi-scale completed local binary patterns and fisher vectors. Remote Sens. 2016, 8, 483. [CrossRef]

35. Jegou, H.; Perronnin, F.; Douze, M.; Sánchez, J.; Pérez, P.; Schmid, C. Aggregating local image descriptors into compact codes. IEEE Trans. Pattern Anal. Mach. Intell. 2011, 34, 1704-1716. [CrossRef]

36. Deng, J.; Dong, W.; Socher, R.; Li, L.; Li, K.; Li, F. ImageNet: A large-scale hierarchical image database. In Proceedings of the IEEE Conference on Computer Vision and Pattern Recognition, Miami, FL, USA, 20-25 June 2009; pp. 248-255.

37. Zhou, W.; Newsam, S.; Li, C.; Shao, Z. Learning low dimensional convolutional neural networks for high-resolution remote sensing image retrieval. Remote Sens. 2017, 9, 489. [CrossRef]

38. Nogueira, K.; Penatti, O.A.; dos Santos, J.A. Towards better exploiting convolutional neural networks for remote sensing scene classification. Pattern Recognit. 2017, 61, 539-556. [CrossRef]

39. Hu, F.; Xia, G.S.; Hu, J.; Zhang, L. Transferring deep convolutional neural networks for the scene classification of high-resolution remote sensing imagery. Remote Sens. 2015, 7, 14680-14707. [CrossRef]

40. Wang, J.; Liu, W.; Ma, L.; Chen, H.; Chen, L. IORN: An effective remote sensing image scene classification framework. IEEE Geosci. Remote Sens. Lett. 2018, 15, 1695-1699. [CrossRef] 
41. Li, J.; Lin, D.; Wang, Y.; Xu, G.; Ding, C. Deep discriminative representation learning with attention map for scene classification. arXiv 2019, arXiv:1902.07967.

42. Chen, Z.; Wang, S.; Hou, X.; Shao, L.; Dhabi, A. Recurrent transformer network for remote sensing scene categorization. In Proceedings of the 29th British Machine Vision Conference, Newcastle, UK, 3-6 September 2018; p. 266.

43. Boualleg, Y.; Farah, M.; Farah, I.R. Remote sensing scene classification using convolutional features and deep forest classifier. IEEE Geosci. Remote Sens. Lett. 2019, 16, 1944-1948. [CrossRef]

44. Xie, J.; He, N.; Fang, L.; Plaza, A. Scale-free convolutional neural network for remote sensing scene classification. IEEE Trans. Geosci. Remote Sens. 2019, 57, 6916-6928. [CrossRef]

45. Wang, Q.; Liu, S.; Chanussot, J.; Li, X. Scene classification with recurrent attention of VHR remote sensing images. IEEE Trans. Geosci. Remote Sens. 2018, 57, 1155-1167. [CrossRef]

46. Guo, Y.; Ji, J.; Lu, X.; Huo, H.; Fang, T.; Li, D. Global-Local attention network for aerial scene classification. IEEE Access 2019, 7, 67200-67212. [CrossRef]

47. Cheng, G.; Yang, C.; Yao, X.; Guo, L.; Han, J. When deep learning meets metric learning: Remote sensing image scene classification via learning discriminative CNNs. IEEE Trans. Geosci. Remote Sens. 2018, 56, 2811-2821. [CrossRef]

48. Wei, T.; Wang, J.; Liu, W.; Chen, H.; Shi, H. Marginal center loss for deep remote sensing image scene classification. IEEE Geosci. Remote Sens. Lett. 2019, 1-5. [CrossRef]

49. Ye, L.; Wang, L.; Zhang, W.; Li, Y.; Wang, Z. Deep metric learning method for high resolution remote sensing image scene classification. Acta Geod. Cartogr. Sin. 2019, 48, 698-707.

50. Goel, A.; Banerjee, B.; Pižurica, A. Hierarchical metric learning for optical remote sensing scene categorization. IEEE Geosci. Remote Sens. Lett. 2018, 16, 952-956. [CrossRef]

51. Anwer, R.M.; Khan, F.S.; van de Weijer, J.; Molinier, M.; Laaksonen, J. Binary patterns encoded convolutional neural networks for texture recognition and remote sensing scene classification. arXiv 2017, arXiv:1706.01171. [CrossRef]

52. Huang, $\mathrm{H} . ; \mathrm{Xu}, \mathrm{K}$. Combing triple-part features of convolutional neural networks for scene classification in remote sensing. Remote Sens. 2019, 11, 1687. [CrossRef]

53. Zhu, Q.; Zhong, Y.; Liu, Y.; Zhang, L.; Li, D. A deep-local-global feature fusion framework for high spatial resolution imagery scene classification. Remote Sens. 2018, 10, 568.

54. Bian, X.; Chen, C.; Tian, L.; Du, Q. Fusing local and global features for high-resolution scene classification. IEEE J. Sel. Top. Appl. Earth Obs. Remote Sens. 2017, 10, 2889-2901. [CrossRef]

55. Zhu, Q.; Zhong, Y.; Zhao, B.; Xia, G.; Zhang, L. Bag-of-visual-words scene classifier with local and global features for high spatial resolution remote sensing imagery. IEEE Geosci. Remote Sens. Lett. 2016, 13, 747-751. [CrossRef]

56. Simonyan, K.; Zisserman, A. Very deep convolutional networks for large-scale image recognition. arXiv 2014, arXiv:1409.1556.

57. Chaib, S.; Liu, H.; Gu, Y.; Yao, H. Deep feature fusion for VHR remote sensing scene classification. IEEE Trans. Geosci. Remote Sens. 2017, 55, 4775-4784. [CrossRef]

58. Szegedy, C.; Liu, W.; Jia, Y.; Sermanet, P.; Reed, S.; Anguelov, D.; Erhan, D.; Vanhoucke, V.; Rabinovich, A. Going deeper with convolutions. In Proceedings of the IEEE Conference on Computer Vision and Pattern Recognition (CVPR'15), Boston, MA, USA, 7-12 June 2015; pp. 1-9.

59. He, K.; Zhang, X.; Ren, S.; Sun, J. Deep residual learning for image recognition. In Proceedings of the IEEE Conference on Computer Vision and Pattern Recognition (CVPR'16), Las Vegas, NV, USA, 26 June-1 July 2016; pp. 770-778.

60. Zhang, B.; Zhang, Y.; Wang, S. A Lightweight and Discriminative Model for Remote Sensing Scene Classification with Multidilation Pooling Module. IEEE J. Sel. Top. Appl. Earth Obs. Remote Sens. 2019, 12, 2636-2653. [CrossRef]

61. Zhang, G.; Lei, T.; Cui, Y.; Jiang, P. A Dual-Path and Lightweight Convolutional Neural Network for High-Resolution Aerial Image Segmentation. ISPRS Int. J. Geo-Inf. 2019, 8, 582. [CrossRef]

62. Teimouri, N.; Dyrmann, M.; Jørgensen, R. A Novel Spatio-Temporal FCN-LSTM Network for Recognizing Various Crop Types Using Multi-Temporal Radar Images. Remote Sens. 2019, 11, 990. [CrossRef] 
63. Lin, T.Y.; RoyChowdhury, A.; Maji, S. Bilinear CNN models for fine-grained visual recognition. In Proceedings of the IEEE International Conference on Computer Vision (ICCV), Santiago, Chile, 7-13 December 2015; pp. 1449-1457.

64. Yu, C.; Zhao, X.; Zheng, Q.; Zhang, P.; You, X. Hierarchical bilinear pooling for fine-grained visual recognition. In Proceedings of the European Conference on Computer Vision (ECCV), Munich, Germany, 8-14 September 2018; pp. 574-589.

65. Sandler, M.; Howard, A.; Zhu, M.; Zhmoginov, A.; Chen, L. Mobilenetv2: Inverted residuals and linear bottlenecks. In Proceedings of the IEEE Conference on Computer Vision and Pattern Recognition, Salt Lake City, UT, USA, 18-22 June 2018; pp. 4510-4520.

66. Howard, A.G.; Zhu, M.; Chen, B.; Kalenichenko, D.; Wang, W.; Weyand, T.; Andreetto, M.; Adam, H. Mobilenets: Efficient convolutional neural networks for mobile vision applications. arXiv 2017, arXiv:1704.04861.

67. Negrel, R.; Picard, D.; Gosselin, P.H. Evaluation of second-order visual features for land-use classification. In Proceedings of the 12th International Workshop on Content-Based Multimedia Indexing (CBMI), Klagenfurt, Austria, 18-20 June 2014; pp. 1-5.

68. Weng, Q.; Mao, Z.; Lin, J.; Guo, W. Land-use classification via extreme learning classifier based on deep convolutional features. IEEE Geosci. Remote Sens. Lett. 2017, 14, 704-708. [CrossRef]

69. Yu, Y.; Liu, F. A two-stream deep fusion framework for high-resolution aerial scene classification. Comput. Intell. Neurosci. 2018, 2018, 1-13. [CrossRef] [PubMed]

70. Liu, N.; Lu, X.; Wan, L.; Huo, H.; Fang, T. Improving the separability of deep features with discriminative convolution filters for RSI classification. ISPRS Int. J. Geo-Inf. 2018, 7, 95. [CrossRef]

71. Liu, X.; Zhou, Y.; Zhao, J.; Yao, R.; Liu, B. Siamese convolutional neural networks for remote sensing scene classification. IEEE Geosci. Remote Sens. Lett. 2019, 16, 1200-1204. [CrossRef]

72. Wang, C.; Lin, W.; Tang, P. Multiple resolution block feature for remote-sensing scene classification. Int. J. Remote Sens. 2019, 40, 6884-6904. [CrossRef]

73. Liu, B.D.; Meng, J.; Xie, W.Y.; Shao, S.; Li, Y.; Wang, Y.J. Weighted spatial pyramid matching collaborative representation for remote-sensing-image scene classification. Remote Sens. 2019, 11, 518. [CrossRef]

74. Zhang, W.; Tang, P.; Zhao, L. Remote sensing image scene classification using CNN-CapsNet. Remote Sens. 2019, 11, 494. [CrossRef] 